\title{
Unquenching the gluon propagator with Schwinger-Dyson equations
}

\author{
A. C. Aguilar, ${ }^{1}$ D. Binosi, ${ }^{2}$ and J. Papavassiliou ${ }^{3}$ \\ ${ }^{1}$ Federal University of $A B C, C C N H$, \\ Rua Santa Adélia 166, CEP 09210-170, Santo André, Brazil. \\ ${ }^{2}$ European Centre for Theoretical Studies in Nuclear Physics \\ and Related Areas (ECT*) and Fondazione Bruno Kessler, \\ Villa Tambosi, Strada delle Tabarelle 286, I-38123 Villazzano (TN) Italy \\ ${ }^{3}$ Department of Theoretical Physics and IFIC, University of Valencia and CSIC, \\ E-46100, Valencia, Spain
}

\begin{abstract}
In this article we use the Schwinger-Dyson equations to compute the nonperturbative modifications caused to the infrared finite gluon propagator (in the Landau gauge) by the inclusion of a small number of quark families. Our basic operating assumption is that the main bulk of the effect stems from the "one-loop dressed" quark loop contributing to the full gluon self-energy. This quark loop is then calculated, using as basic ingredients the full quark propagator and quark-gluon vertex; for the quark propagator we use the solution obtained from the quark gap equation, while for the vertex we employ suitable Ansätze, which guarantee the transversality of the answer. The resulting effect is included as a correction to the quenched gluon propagator, obtained in recent lattice simulations. Our main finding is that the unquenched propagator displays a considerable suppression in the intermediate momentum region, which becomes more pronounced as we increase the number of active quark families. The influence of the quarks on the saturation point of the propagator cannot be reliably computed within the present scheme; the general tendency appears to be to decrease it, suggesting a corresponding increase in the effective gluon mass. The renormalization properties of our results, and the uncertainties induced by the unspecified transverse part of the quark-gluon vertex, are discussed. Finally, the dressing function of the gluon propagator is compared with the available unquenched lattice data, showing rather good agreement.
\end{abstract}

PACS numbers: 12.38.Aw, 12.38.Lg, 14.70.Dj 


\section{INTRODUCTION}

In recent years considerable progress has been made in our understanding of various aspects of the nonperturbative dynamics of Yang-Mills theories, through the fruitful combination of a variety of approaches and techniques [1-28]. Particularly successful in this effort has been the continuous interplay between lattice simulations and Schwinger-Dyson equations (SDEs) [29 34], which has led to a firmer grasp on the infrared (IR) behavior of the fundamental Green's functions of QCD, such as gluon, ghost, and quark propagators, as well as some of the basic vertices of the theory, for special kinematic configurations [35-37].

A significant part of the existing SDE analysis has focused on the study of various aspect of the aforementioned Green's functions at the level of pure gauge Yang-Mills theories, i.e., without the inclusion of quarks [15, 21, 25]. This tendency has been mainly motivated by the fact that the vast majority of lattice simulations work in the quenched limit, making no reference to effects stemming from dynamical quarks [1, 7, 9].

The transition from pure $S U(3)$ Yang-Mills Green's functions to those of real world QCD is, of course, highly nontrivial, and has been the focal point of relatively few lattice investigations [5, 6]. At the level of the SDEs, to the best of or knowledge, this issue has been studied in detail [38, 39] only in the context of the so-called "scaling solutions" [12], but no analogous investigation has been carried out for the (IR finite) massive solutions [40], found both in the lattice simulations and in several of the analytic studies cited above.

The purpose of the present article is to provide a self-consistent framework for addressing this latter problem in the continuum, at the level of the corresponding SDEs. In particular, we will present an approximate method for "unquenching" the (IR finite) gluon propagator (in the Landau gauge), computing nonperturbatively the effects induced by a small number of light quark families.

The method we present consists of two basic steps: $(i)$ computing the fully-dressed quark-loop diagram [see graph $\left(a_{11}\right)$ in Fig. 1], using as input the nonperturbative quark propagators obtained from the solution of the gap equation, together with an Ansatz for the fully-dressed quark-gluon vertex that preserves gauge-invariance [31]; and (ii) adding the result computed in $(i)$ to the quenched gluon propagator obtained in the large-volume lattice simulations mentioned above [7]. The key assumption of the method employed is that the effects of a small number of quark families to the gluon propagator may be considered as 
a "perturbation" to the quenched case, and that the diagram $\left(a_{11}\right)$ constitutes the leading correction. The subleading corrections stem from the (originally) pure Yang-Mills diagrams [graphs $\left(a_{1}\right)-\left(a_{10}\right)$ in Fig. [1, which now get modified from the quark loops nested inside them (see Fig. 3); their proper inclusion, however, lies beyond our present calculation powers. So, our operating assumption is that these latter effects are small compared to those originating from graph $\left(a_{11}\right)$, and will be neglected at this level of approximation. It is interesting to note that in the context of the "scaling" solutions this latter assumption appears to be indeed reasonable [38, 39].

This assumption becomes relevant when implementing point (ii), where the contributions from graphs $\left(a_{1}\right)-\left(a_{10}\right)$ will be taken to be exactly the same as those of the quenched case even when dynamical quarks are present, thus identifying with the quenched lattice propagator everything except graph $\left(a_{11}\right)$. Of course, as is typical in the SDE studies, the validity of this central assumption may be tested only a-posteriori, either by means of additional, more complicated computations, or, more pragmatically, through the levels of agreement achieved with available lattice results. As we will see in the main body of the article [Section [V-D], the general features emerging from our calculations are consistent with the lattice results of [5, 6].

The general framework we will adopt is based on the synthesis of the the pinch technique (PT) [16, 40-43] with the background field method (BFM) [44], known in the literature as the PT-BFM scheme [13, 14, 45]. As has been explained in detail in various works, the PT-BFM Green's functions satisfy Abelian-like Ward identities (WIs), instead of the typical Slavnov-Taylor identities (STIs), valid within the linear covariant $\left(R_{\xi}\right)$ gauges [16, 44]. The main consequence of this property is that the resulting SDE for the gluon self-energy may be suitably truncated, without compromising the transversality of the answer [13, 14, 45].

For the case at hand, the new ingredient is the nonperturbative quark loop, which is transverse in the PT-BFM scheme as well as in the $R_{\xi}$ gauges; thus, at first sight, it would seem that there is no real advantage in using the former scheme. However, the important issue at this point is the exact way how this transversality is realized in both cases. In particular, the fact that the fully dressed quark-gluon vertex of the PT-BFM (denoted by $\widehat{\Gamma}_{\mu}$ ) satisfies a QED-like WI provides a definite advantage over the corresponding conventional vertex (denoted by $\Gamma_{\mu}$ ), which satisfies the STI that involves the quark-ghost scattering kernel [46], a relatively unexplored quantity (see Eqs. (3.4) and (3.3), respectively) [31]. 
The reason why this constitutes an advantage has to do with the fact that, according to the common practice, one must eventually introduce a suitable nonperturbative Ansatz for the full quark-gluon vertex, such that the corresponding WIs (or STIs) are automatically satisfied. The fact that the PT-BFM vertex satisfies a WI instead of an STI simplifies the problem considerably, because it allows one to employ the time-honored Abelian Ansätze existing in the literature [47, 48].

The necessary transition from the PT-BFM to the conventional gluon propagator, which is the one simulated on the lattice, is accomplished by means of a special Green's function, usually denoted by $G$ in the literature [14, 45]. In the Landau gauge, $G$ is known to coincide with the "Kugo-Ojima" function, and to be related to the ghost dressing function by means of a powerful identity enforced by the underlying Becchi-Rouet-Stora-Tyutin (BRST) symmetry [49, 50]. Thus, the use of the PT-BFM scheme eliminates the need to refer to quantities such as the quark-ghost kernel, at the very modest price of introducing the aforementioned function, which, due to its STI, can be accurately reconstructed from large-volume lattice data on the ghost dressing functions [50, 51], or possibly through direct lattice simulations of the Kugo-Ojima function [52].

The main results of our study may be summarized as follows. The basic effect of the quark loop(s) (one or two families with a constituent mass of the order of $300 \mathrm{MeV}$ ) is to suppress considerably the gluon propagator in the IR and intermediate momenta regions, while the ultraviolet (UV) tails increase, exactly as expected from the standard renormalization group analysis. The final saturation point of the unquenched propagator cannot be reliably calculated at present; the apparent tendency is that the inclusion of light quarks makes the gluon propagator saturate at a lower point, which can be translated into having a larger gluon mass. We emphasize that the way the quark loops affect the value of the gluon mass is indirect: the contribution obtained from graph $\left(a_{11}\right)$ vanishes at $q^{2}=0$, so it does not change the gluon mass equation formally [53]; however, it does change its solutions, because of the modification that it induces in the intermediate region of the gluon propagator (which enters in the gluon mass equation). A reliable estimate of this gluon mass difference cannot be obtained without resorting to the full gluon mass equation, whose derivation is currently underway. For the purposes of the present work, the IR "saturation point" of the unquenched propagator will be estimated only approximately, through a processes of "extrapolation" of the intermediate momenta region towards the deep IR. The dependence 
of the results on the renormalization point $\mu$ is also studied in detail, and appears to be consistent with expectations based on general considerations.

In addition, we present a direct comparison between unquenched gluon "dressing functions", namely the one obtained using the method described above with that found on the lattice [5, 6]. Note that, due to its very definition, the dressing function is rather insensitive to the exact value of the final saturation point, moderating to some extent the effect of the aforementioned uncertainty. The resulting comparison with the lattice data is rather favorable, as may be seen in Fig. 17, in the momentum region of maximum discrepancy the two curves differ by about 10\%, being significantly closer everywhere else.

Finally, it is quite interesting to mention that the use of the perturbative result for the quark loop [see Appendix] gives rise to an effect that is numerically very close to that obtained through the more sophisticated field-theoretic treatment described above, as can be appreciated on the left panel of Fig. 15,

The article is organized as follows. In section II] we give a detailed presentation of the basic methodology, main ingredients, and central assumptions of the procedure employed for adding quark loops to the gluon propagator. In section III we elaborate on the way how the quark loop is computed nonperturbatively. The main points of this section include $(i)$ the particular form(s) of the full quark-gluon vertex employed, (ii) the actual computation of the loop and its behavior at $q^{2}=0,($ iii $)$ the (subtractive) renormalization procedure, and $(i v)$ the transition to the Euclidean space. Section [V] contains the main results of the present work. After introducing the lattice ingredients used as input in our basic formulas, we present the unquenched gluon propagator for $S U(3)$, together with the corresponding dressing function, for a small number of light quark families. Further relevant points, such as the dependence of the results on the renormalization point, as well as the effect of "decoupling" of the heavy quarks are also addressed. In addition, a comparison of the resulting gluon dressing function with available lattice data [5, 6] is presented. Our main conclusions and further open questions are summarized in section $\mathrm{V}$. Finally, some useful formulas related to the perturbative (one-loop) calculation of the quark loop are summarized in an Appendix. 


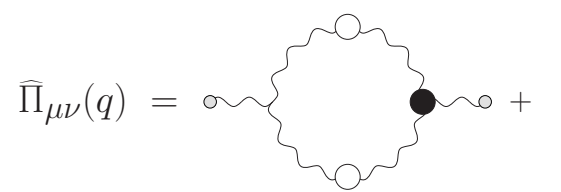

$\left(a_{1}\right)$

$\left(a_{5}\right)$

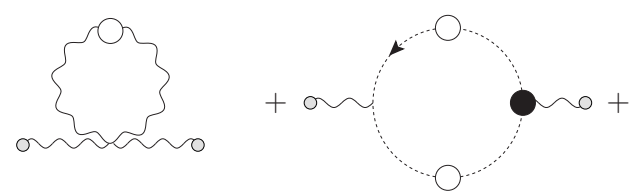

$\left(a_{3}\right)$

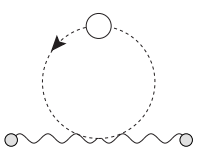

$\left(a_{4}\right)$
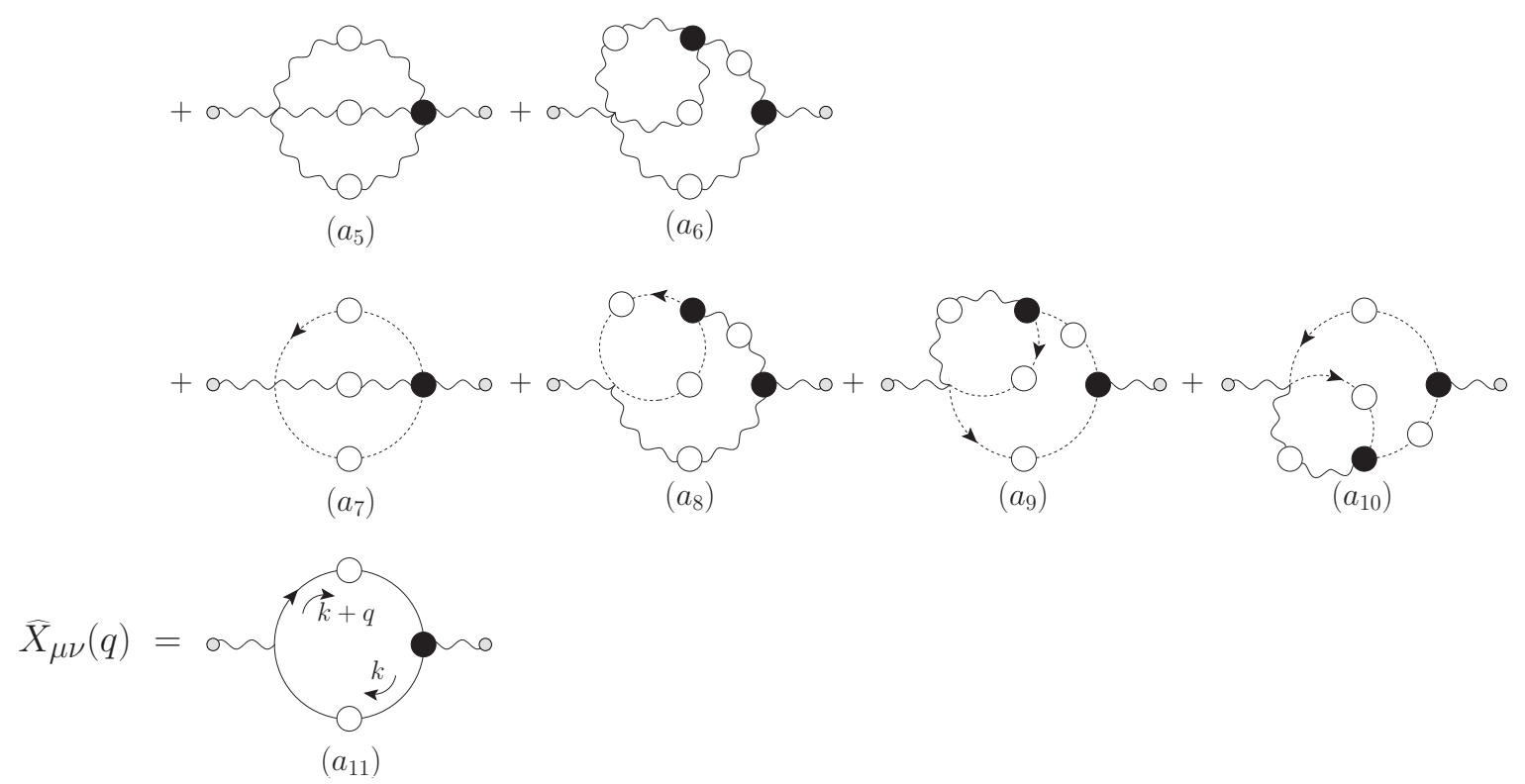

FIG. 1: The full PT-BFM gluon self-energy. White (respectively black) blobs represents connected (respectively 1-particle irreducible) Green's functions; the small gray circles on the external legs indicate background gluons.

\section{ADDING QUARK LOOPS TO THE GLUON PROPAGATOR}

To begin with, in the Landau gauge the gluon propagator (quenched or unquenched) assumes the form

$$
\Delta_{\mu \nu}(q)=-i \Delta\left(q^{2}\right) P_{\mu \nu}(q) ; \quad P_{\mu \nu}(q)=g_{\mu \nu}-\frac{q_{\mu} q_{\nu}}{q^{2}}
$$

Let us now denote by $\Delta_{Q}\left(q^{2}\right)$ the full gluon propagator in the presence of quark loops, while the corresponding quenched propagator, i.e., the full gluon propagator in the absence of quark loops, will be denoted simply by $\Delta\left(q^{2}\right)$.

In the PT-BFM scheme, $\Delta\left(q^{2}\right)$ satisfies the following SDE [14-16],

$$
\Delta^{-1}\left(q^{2}\right) P^{\mu \nu}(q)=\frac{q^{2} P^{\mu \nu}(q)+i \widehat{\Pi}^{\mu \nu}(q)}{\left[1+G\left(q^{2}\right)\right]^{2}} .
$$




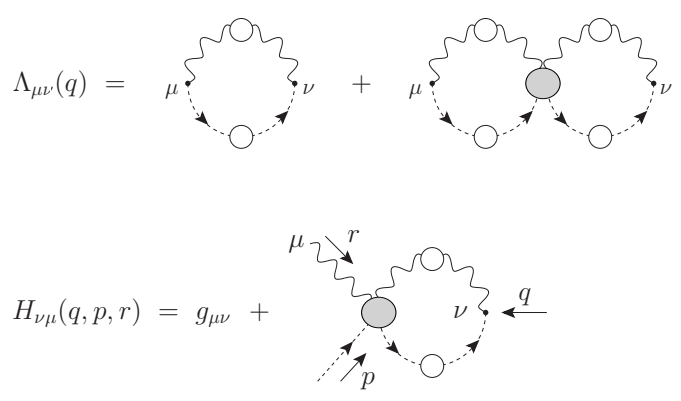

FIG. 2: Definitions and conventions of the auxiliary functions $\Lambda$ and $H$. The color and gauge coupling dependence for the field combination shown, $c^{a}(p) A_{\mu}^{b}(r) A_{\nu}^{* c}(q)$, is $g f^{a c b}$. Gray blobs denote one-particle irreducible (with respect to vertical cuts) Schwinger-Dyson kernels.

where

$$
\widehat{\Pi}^{\mu \nu}(q)=\sum_{i=1}^{10}\left(a_{i}\right)^{\mu \nu},
$$

and the relevant fully dressed diagrams $\left(a_{i}\right)$ are shown in Fig. 1, All these diagrams contain only fields appearing in the pure gauge Yang-Mills Lagrangian, namely gluons and ghosts. The function $G$ appearing in (2.2) is particular to the PT-BFM formalism [14, 45]; specifically, it is the form factor associated with the metric tensor $g_{\mu \nu}$ in the Lorentz decomposition of the auxiliary two-point function $\Lambda_{\mu \nu}$, given by [16]

$$
\begin{aligned}
\Lambda_{\mu \nu}(q) & =-i g^{2} C_{A} \int_{k} \Delta_{\mu}^{\sigma}(k) D(q-k) H_{\nu \sigma}(-q, q-k, k) \\
& =g_{\mu \nu} G\left(q^{2}\right)+\frac{q_{\mu} q_{\nu}}{q^{2}} L\left(q^{2}\right) .
\end{aligned}
$$

In the formula above, $C_{A}$ is the Casimir eigenvalue in the adjoint representation $\left[C_{A}=N\right.$ for $S U(N)$ ], and the $d$-dimensional integral (in dimensional regularization) is defined according to

$$
\int_{k} \equiv \frac{\mu^{\epsilon}}{(2 \pi)^{d}} \int \mathrm{d}^{d} k
$$

with $d=4-\epsilon$ and $\mu$ the 't Hooft mass. The function $\Lambda_{\mu \nu}(q)$, together with the auxiliary function $H_{\mu \nu}(q, p, r)$, are diagrammatically represented in Fig. 2 .

Notice that $H_{\mu \nu}$ is related to the (conventional) gluon-ghost $\Gamma_{\mu}^{c}$ vertex by the identity

$$
p^{\nu} H_{\nu \mu}(p, r, q)+\Gamma_{\mu}^{c}(r, q, p)=0
$$

and that, in the (background) Landau gauge, the following all order relation holds [49, 50]

$$
F^{-1}\left(q^{2}\right)=1+G\left(q^{2}\right)+L\left(q^{2}\right)
$$




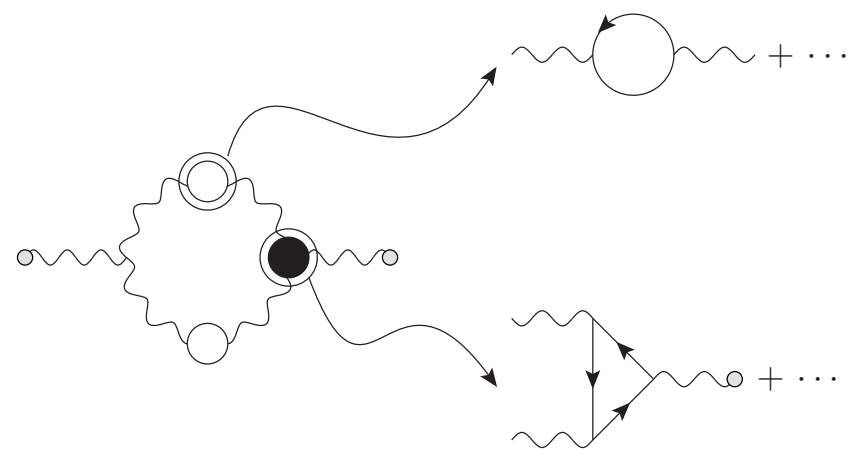

FIG. 3: The nonlinear propagation of the effect of unquenching the gluon propagator through the addition of dynamical fermions, shown here for the one-loop dressed gluon diagram $\left(a_{1}\right)$. Both the internal gluon propagator and the three-gluon vertex gets modified (shown here by two representative graphs only); similar modifications occur for all other diagrams.

The unquenched propagator in the presence of a single quark loop will satisfy an appropriately modified version of (2.2), namely

$$
\Delta_{Q}^{-1}\left(q^{2}\right) P^{\mu \nu}(q)=\frac{q^{2} P^{\mu \nu}(q)+i \widehat{\Pi}_{Q}^{\mu \nu}(q)+i \widehat{X}^{\mu \nu}(q)}{\left[1+G_{Q}\left(q^{2}\right)\right]^{2}} .
$$

The main difference between (2.2) and (2.8) is the explicit appearance of $\widehat{X}^{\mu \nu}(q)$ on the rhs, originating entirely from diagram $\left(a_{11}\right)$ (see again Fig. 1). Notice, however, that there will be a nonlinear propagation of the changes induced due to $\widehat{X}^{\mu \nu}(q)$, which will also affect the original subset of purely Yang-Mills graphs, namely $\left(a_{1}\right)-\left(a_{10}\right)$, given that now the various Green's functions appearing inside them will have been modified by $\widehat{X}^{\mu \nu}(q)$. For example, at the "one-loop dressed" level, diagram $\left(a_{1}\right)$ receives quark-loop contributions, such as those shown in Fig. 3, and the same happens with all other graphs belonging to the set $\left(a_{1}\right)-\left(a_{10}\right)$. This complicated nonlinear effect is indicated by introducing the suffix $Q$ in the associated self-energy, $\widehat{\Pi}_{Q}^{\mu \nu}(q)$. The quantity $G(q)$ will be similarly affected by the inclusion of the quark loop, as indicated in (2.8) through the substitution $G(q) \rightarrow G_{Q}(q)$. In the case of including various quark loops, corresponding to different quark flavors, $Q_{i}$, the term $\widehat{X}^{\mu \nu}(q)$ in (2.8) is replaced simply by the sum over all quark loops, i.e.,

$$
\widehat{X}^{\mu \nu}(q) \rightarrow \sum_{i} \widehat{X}_{i}(q)
$$

The tensorial structure in Eqs. (2.2) and (2.8) may be easily eliminated, by appealing to the 
transversality properties of the quantities involved on the rhs, namely

$$
q_{\mu} \widehat{\Pi}^{\mu \nu}(q)=0 ; \quad q_{\mu} \widehat{X}^{\mu \nu}(q)=0 ; \quad q_{\mu} \widehat{\Pi}_{Q}^{\mu \nu}(q)=0
$$

Let us now define the scalar cofactors of these quantities as

$$
\widehat{\Pi}^{\mu \nu}(q)=P^{\mu \nu}(q) \widehat{\Pi}\left(q^{2}\right) ; \quad \widehat{X}^{\mu \nu}(q)=P^{\mu \nu}(q) \widehat{X}\left(q^{2}\right) ; \quad \widehat{\Pi}_{Q}^{\mu \nu}(q)=P^{\mu \nu}(q) \widehat{\Pi}_{Q}\left(q^{2}\right) .
$$

Then, equations (2.2) and (2.8) can be converted to their scalar versions, namely

$$
\Delta^{-1}\left(q^{2}\right)=\frac{q^{2}+i \widehat{\Pi}\left(q^{2}\right)}{\left[1+G\left(q^{2}\right)\right]^{2}},
$$

and

$$
\Delta_{Q}^{-1}\left(q^{2}\right)=\frac{q^{2}+i \widehat{\Pi}_{Q}\left(q^{2}\right)+i \widehat{X}\left(q^{2}\right)}{\left[1+G_{Q}\left(q^{2}\right)\right]^{2}} .
$$

Eq. (2.13) can be then straightforwardly adjusted to include the case of various quark loops, simply by replacing $\widehat{X}(q) \rightarrow \sum_{i} \widehat{X}_{Q_{i}}(q)$.

To be sure, the total effect of including quark loops cannot be exactly computed at the level of the SDE, because that would entail the full numerical treatment of the entire series, a task that is beyond our present powers. The way we will proceed instead is the following. We will use the quenched propagator as our reference, and we will estimate the modifications introduced to it by the presence of the quark loop(s), under certain simplifying assumptions that we will now explain.

To that end, let us cast the quenched gluon propagator $\Delta\left(q^{2}\right)$ into the standard form employed in the recent literature [53 55], which incorporates the crucial feature of IR finiteness, implemented by the presence of a dynamically generated gluon mass; specifically, we set (in Minkowski space),

$$
\Delta^{-1}\left(q^{2}\right)=q^{2} J\left(q^{2}\right)-m^{2}\left(q^{2}\right)
$$

The first term on the rhs of (2.14) corresponds to the "kinetic term", or "wave function" contribution, whereas the second is the momentum-dependent mass (which is positive-definite in Euclidean space) [53 55]. As $q^{2} \rightarrow 0$, we have that $q^{2} J_{m}\left(q^{2}\right) \rightarrow 0$; on the other hand, $m^{2}(0) \neq 0$, and as a result, the gluon propagator is IR finite, $\Delta^{-1}(0) \neq 0$. The exact determination of the components $J\left(q^{2}\right)$ and $m^{2}\left(q^{2}\right)$ in terms of the quantities appearing on the rhs of (2.2) and (2.8) is a complicated task, leading eventually to a set of intricate coupled 
integral equations. This exercise has been carried out partially, within the one-loop truncated version of the SDE, considering only the corresponding subset of gluonic contributions [i.e., diagrams $\left(a_{1}\right)$ and $\left.\left(a_{2}\right)\right]$ [53].

In what follows we will operate under the reasonable assumption that the IR finiteness of the gluon propagator persists in the presence of a relatively small number of quark loops. In other words, we assume that the inclusion of two light quark flavors (up and down type quarks, with constituent masses of about $300 \mathrm{MeV}$ ) will affect but not completely destabilize the mechanism responsible for the generation of a dynamical gluon mass, and that their effect may be considered as a "perturbation" to the quenched case. In the realistic case of QCD, the inclusion of loops containing the remaining heavier quarks is expected to give rise to numerically suppressed contributions (compared to those coming from the light quark loops), consistent with the notion of decoupling; this expectation is in fact clearly confirmed in the results presented in Section IV (see in particular Fig. 15). Instead, the theoretical possibility of increasing the number of loops containing light flavors may lead to effects that cannot be longer considered as a "perturbation" of the quenched case: ten families of light quarks, for example, could alter severely the qualitative behavior of the theory, and as a result, the quenched propagator may have little to do with the unquenched one (for a general discussion on how the IR and UV properties of Yang-Mills theories may be distorted, depending on the number of quark families, see, e.g., [56, 57], and references therein).

Thus, under the aforementioned assumptions, Eq. (2.14) will be extended to the case of $\Delta_{Q}\left(q^{2}\right)$, namely

$$
\Delta_{Q}^{-1}\left(q^{2}\right)=q^{2} J_{Q}\left(q^{2}\right)-m_{Q}^{2}\left(q^{2}\right)
$$

where the suffix $Q$ in the dynamical gluon mass indicates the possible modifications to $m^{2}\left(q^{2}\right)$ induced by the quark loop(s), as alluded above. It is important to emphasize that $m^{2}\left(q^{2}\right)$ will change, despite the fact that the main additional ingredient that distinguishes (2.12) and (2.13), namely $\widehat{X}(q)$, does not contribute at $q^{2}=0$, since $\widehat{X}(0)=0$ [see Eq. (3.19)], and therefore it does not affect directly the gluon mass equation [53]; instead, the modification induced is indirect, due to the change in the overall shape of $\Delta\left(q^{2}\right)$ throughout the entire range of momenta. In order to gain a qualitative understanding of this last statement, let us consider the IR limit of the approximate gluon mass equation obtained in [53], where only 
the one-loop dressed graphs $\left(a_{1}\right)$ and $\left(a_{2}\right)$ are considered; in Euclidean space,

$$
m^{2}(0)=-\frac{3 C_{A}}{8 \pi} \alpha_{s} F(0) \int_{0}^{\infty} \mathrm{d} y m^{2}(y)\left[\mathcal{Z}^{2}(y)\right]^{\prime}+\ldots,
$$

where $\alpha_{s}=g^{2} / 4 \pi$, the prime indicates differentiation with respect to $y=k^{2}$, and $\mathcal{Z}(y)$ is the "dressing function" of the gluon propagator, defines as

$$
\mathcal{Z}\left(q^{2}\right) \equiv q^{2} \Delta\left(q^{2}\right)
$$

Evidently, $\mathcal{Z}(0)=0$. Finally, the ellipses on the rhs of Eq. (2.16) denote contributions from "two-loop dressed" diagrams that have yet to be worked out.

Now, in the presence of quark loops, Eq. (2.16) maintains its functional form, since, as mentioned above, $\widehat{X}(0)=0$; however, the various quantities appearing on its rhs [most notably $\mathcal{Z}(y)$ ] will be modified, therefore acquiring a suffix "Q" $\left[\right.$ e.g., $\left.\mathcal{Z}(y) \rightarrow \mathcal{Z}_{Q}(y)\right]$. As a consequence, the resulting solution gets modified, and we have $m^{2}\left(q^{2}\right) \rightarrow m_{Q}^{2}\left(q^{2}\right)$; in what follows we will denote by

$$
\lambda^{2} \equiv m_{Q}^{2}(0)-m^{2}(0)
$$

the gluon mass difference at $q^{2}=0$.

As already explained, a solid first-principle determination of $\lambda^{2}$ is not possible at the moment, mainly due to the fact that the available gluon mass equation (2.16) is incomplete, since it has been derived from only one subset of the relevant graphs [53]. Therefore, in the analysis presented we will restrict ourselves to extracting an approximate range for $\lambda^{2}$, through the extrapolation of the curves obtained from intermediate momenta towards the deep IR.

In order to estimate the effect of the quark $\operatorname{loop}(\mathrm{s})$ on the gluon propagator, we will assume that the main bulk of the correction to the "kinetic" part, $q^{2} J_{Q}\left(q^{2}\right)$, is due to the direct presence of the extra diagram $\left(a_{11}\right)$. Instead, the nonlinear effect due to the fact that the graphs $\left(a_{1}\right)-\left(a_{10}\right)$ develop an indirect quark dependence, i.e., $\widehat{\Pi}\left(q^{2}\right) \rightarrow \widehat{\Pi}_{Q}\left(q^{2}\right)$, is predominantly responsible for the change in the gluon mass, as captured in (2.18), inducing minor changes to the kinetic part $q^{2} J_{Q}\left(q^{2}\right)$. Finally, we will approximate the function $G_{Q}\left(q^{2}\right)$ appearing in the denominator of Eq. (2.13) by the quenched expression, i.e., $G_{Q}\left(q^{2}\right) \rightarrow G\left(q^{2}\right)$; as can be seen from its defining equation Eq. (2.4) and Fig. 2, quark-loops enter only as "higher order" effects, according to our general philosophy, and their effect should be small. 
Thus, within this approximation scheme, the quantity $J_{Q}\left(q^{2}\right)$ will be given by

$$
q^{2} J_{Q}\left(q^{2}\right)=q^{2} J\left(q^{2}\right)+\frac{i \widehat{X}\left(q^{2}\right)}{\left[1+G\left(q^{2}\right)\right]^{2}} .
$$

If we now combine Eqs. (2.14), (2.15), (2.16) and (2.18), it is easy to arrive at the result (Minkowski space)

$$
\Delta_{Q}\left(q^{2}\right)=\frac{\Delta\left(q^{2}\right)}{1+\left\{i \widehat{X}\left(q^{2}\right)\left[1+G\left(q^{2}\right)\right]^{-2}-\lambda^{2}\right\} \Delta\left(q^{2}\right)} .
$$

In what follows we will identify the quenched propagator $\Delta\left(q^{2}\right)$ appearing on the rhs of (2.20) with the one obtained from the large volume lattice simulations [7], to be denoted by $\Delta_{L}\left(q^{2}\right)$. So, effectively one assumes that $\Delta_{L}\left(q^{2}\right)$ is a solution of the full SDE equation, with no quarks, given in (2.12); thus, when using (2.20) we will be carrying out the replacement $\Delta\left(q^{2}\right) \rightarrow \Delta_{L}\left(q^{2}\right)$.

\section{NONPERTURBATIVE QUARK LOOP IN THE PT-BFM SCHEME}

In this section we present the actual nonperturbative calculation of the quark-loop diagram $\left(a_{11}\right)$, finally expressing the answer exclusively in terms of the functions $A(p)$ and $B(p)$, appearing in the Dirac decomposition of the full quark propagator [see (3.7)]. The calculation relies on the use of suitable Ansätze for the fully dressed quark-gluon vertex appearing in $\left(a_{11}\right)$, presented and discussed in the corresponding subsection. The Euclidean version of the (renormalized) master formula that we use in the next section in order to estimate the effect of the quark loop on the gluon propagator is given in Eq. (3.33).

\section{A. The quark-gluon vertex}

The quantity responsible for the difference between the quark loop in the conventional covariant gauges and the PT-BFM scheme is the fully-dressed quark-gluon vertex. Specifi-

cally, let us denote the fully dressed PT-BFM quark-gluon vertex by $\widehat{\mathbb{\Gamma}}_{\mu}^{a}$, and factor out the color structure, according to

$$
\widehat{\mathbb{\Gamma}}_{\mu}^{a}\left(p_{1}, p_{2}, p_{3}\right)=g t^{a} \widehat{\Gamma}_{\mu}\left(p_{1}, p_{2}, p_{3}\right),
$$




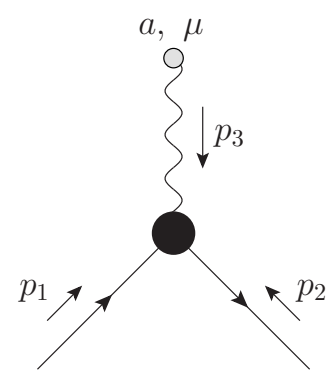

FIG. 4: The full PT-BFM quark-gluon vertex $\widehat{\mathbb{\Gamma}}_{\mu}^{a}$. Note that $p_{1} \leftrightarrow p_{2}$ with respect to the conventions used in [31, 58].

where all momenta $p_{i}$ entering (see Fig. 4); at tree-level, $\widehat{\Gamma}_{\mu}^{(0)}=\gamma_{\mu}$. In the equation above, $t^{a}$ represents the $N^{2}-1$ hermitian and traceless generators of the $S U(N)$ gauge group, satisfying the algebra

$$
\left[t^{a}, t^{b}\right]=i f^{a b c} t^{c}
$$

with $f^{a b c}$ the totally antisymmetric group structure constants. In the $S U(3)$ case, with the quarks in the fundamental representation, $t^{a}=\lambda^{a} / 2$, where $\lambda^{a}$ are the Gell-Mann matrices.

In the conventional formulation within the linear covariant gauges, the quark-gluon vertex, to be denoted by $\Gamma_{\mu}\left(p_{1}, p_{2}, p_{3}\right)$, satisfies the well-known STI [46]

$$
i p_{3}^{\mu} \Gamma_{\mu}\left(p_{1}, p_{2}, p_{3}\right)=F\left(p_{3}\right)\left[S^{-1}\left(p_{1}\right) H\left(p_{2}, p_{1}, p_{3}\right)-\bar{H}\left(p_{1}, p_{2}, p_{3}\right) S^{-1}\left(-p_{2}\right)\right]
$$

where $S^{-1}(p)$ is the inverse of the full quark propagator, $H\left(p_{2}, p_{1}, p_{3}\right)$ is the quark-ghost scattering kernel diagrammatically defined in Fig. 5, and $\bar{H}\left(p_{1}, p_{2}, p_{3}\right)$ its "conjugate".

In contrast, in the PT-BFM scheme, the vertex $\widehat{\Gamma}_{\mu}$ satisfies the QED-like WI [16, 44]

$$
i p_{3}^{\mu} \widehat{\Gamma}_{\mu}\left(p_{1}, p_{2}, p_{3}\right)=S^{-1}\left(p_{1}\right)-S^{-1}\left(-p_{2}\right)
$$

with no reference whatsoever to the ghost sector. Then, the most general Ansatz for the longitudinal part of $\widehat{\Gamma}_{\mu}$ that satisfies (3.4) is given by [47]

$$
\widehat{\Gamma}_{\mu}\left(p_{1}, p_{2}, p_{3}\right)=L_{1}\left(p_{1}, p_{2}\right) \gamma_{\mu}+L_{2}\left(p_{1}, p_{2}\right)\left(\not p_{1}-\not p_{2}\right)\left(p_{1}-p_{2}\right)_{\mu}+L_{3}\left(p_{1}, p_{2}\right)\left(p_{1}-p_{2}\right)_{\mu} .
$$

The form factors $L_{i}$ appearing in the expression above are given by

$$
L_{1}\left(p_{1}, p_{2}\right)=\frac{A\left(p_{1}\right)+A\left(p_{2}\right)}{2} ; \quad L_{2}\left(p_{1}, p_{2}\right)=\frac{A\left(p_{1}\right)-A\left(p_{2}\right)}{2\left(p_{1}^{2}-p_{2}^{2}\right)} ; \quad L_{3}\left(p_{1}, p_{2}\right)=-\frac{B\left(p_{1}\right)-B\left(p_{2}\right)}{p_{1}^{2}-p_{2}^{2}} .
$$




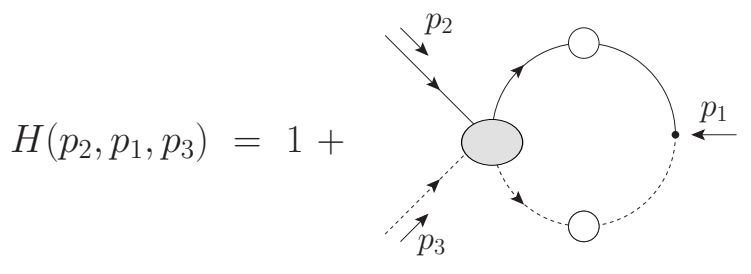

FIG. 5: Diagrammatic representation of the quark-ghost scattering kernel $H\left(p_{1}, p_{2}, p_{3}\right)$.

where the functions $A(p)$ and $B(p)$ are defined as

$$
S^{-1}(p)=-i[A(p) \not p-B(p)]=-i A(p)[\not p-\mathcal{M}(p)]
$$

and the ratio $\mathcal{M}(p)=B(p) / A(p)$ is the dynamical quark mass function. For latter convenience, we will denote the dynamical quark mass function at $p^{2}=0$ by $\mathcal{M}(0) \equiv M$. Therefore, at tree level $(A=1, B=M)$ and one has $L_{1}=1$ and $L_{2}=L_{3}=0$. The resulting vertex reads

$$
\begin{aligned}
\widehat{\Gamma}_{\mu}\left(p_{1}, p_{2}, p_{3}\right) & =\frac{A\left(p_{1}\right)+A\left(p_{2}\right)}{2} \gamma^{\mu} \\
& +\frac{\left(p_{1}-p_{2}\right)^{\mu}}{p_{1}^{2}-p_{2}^{2}}\left\{\left[A\left(p_{1}\right)-A\left(p_{2}\right)\right] \frac{\not p_{1}-\not p_{2}}{2}+\left[B\left(p_{1}\right)-B\left(p_{2}\right)\right]\right\}
\end{aligned}
$$

and is known in the literature as the Ball-Chiu (BC) vertex [47].

We emphasize that in the context of the PT-BFM the longitudinal part of the above vertex is complete, as far as the WI it satisfies is concerned. Indeed, the expression in (3.8) satisfies the exact WI that $\widehat{\Gamma}_{\mu}$ is supposed to obey, namely (3.4). As is well-known, the BC vertex has been employed extensively in the literature (especially in studies of chiral symmetry breaking) [59] as an approximate (denominated "abelianized") version of the conventional $\Gamma_{\mu}$ defined in the covariant gauges. Indeed, the fully dressed quark-gluon vertex entering into the quark gap equation is $\Gamma_{\mu}$ and not $\widehat{\Gamma}_{\mu}$, for the simple reason that the corresponding gluon is quantum and not background; indeed, the gluon in the quark gap equation is internal (i.e., it is irrigated by the virtual momenta), in contrast to the gluon of the quark loop, which is external (carries physical momentum). Therefore, use of the expression given in (3.8) into the quark gap equation constitutes only an approximation, since it fails to satisfy the full STI (3.3) that $\Gamma_{\mu}$ should obey, unless the corresponding ghost sector is turned off.

Note that the $\mathrm{BC}$ vertex has been generalized accordingly in [31], in order to fulfill the exact STI (3.3), thus justifying its use inside the quark gap equation. The corresponding $L_{i}$ 
are considerably more complicated than those given in (3.6), involving the ghost dressing function $F$ and the various form factors of the quark-ghost kernel $H\left(p_{1}, p_{2}, p_{3}\right)$ [31]. In fact, an additional fourth form factor, $L_{4}$, makes its appearance in the Lorentz expansion corresponding to (3.5), multiplying $\sigma_{\mu \nu}=i / 2\left[\gamma_{\mu}, \gamma_{\nu}\right]$; it is then easy to verify that this latter, genuinely non-Abelian vertex of [31] reduces to that of (3.8) in the limit of a trivial ghost sector, i.e., by setting $F(p)=1$ and $H=1$.

Finally, let us comment on an alternative form of the quark-gluon vertex $\widehat{\Gamma}_{\mu}\left(p_{1}, p_{2}, p_{3}\right)$, known in the literature as the Curtis and Pennington (CP) vertex [48], to be denoted by $\widehat{\Gamma}_{\mu}^{\mathrm{CP}}$. This latter vertex satisfies also the WI of (3.4), and differs from the vertex of (3.8) by a transverse (automatically conserved) contribution, which improves its properties under multiplicative renormalizability. Specifically,

$$
\widehat{\Gamma}_{\mu}^{\mathrm{CP}}\left(p_{1}, p_{2}, p_{3}\right)=\widehat{\Gamma}_{\mu}\left(p_{1}, p_{2}, p_{3}\right)+\left[\gamma_{\mu}\left(p_{2}^{2}-p_{1}^{2}\right)+\left(p_{2}-p_{1}\right)_{\mu} p_{3}\right] \widehat{\Gamma}_{\mathrm{T}}\left(p_{1}, p_{2}, p_{3}\right)
$$

where

$$
\widehat{\Gamma}_{\mathrm{T}}\left(p_{1}, p_{2}, p_{3}\right)=\frac{\left[A\left(p_{2}\right)-A\left(p_{1}\right)\right]\left(p_{1}^{2}+p_{2}^{2}\right)}{2\left\{\left(p_{2}^{2}-p_{1}^{2}\right)^{2}+\left[\mathcal{M}^{2}\left(p_{2}\right)+\mathcal{M}^{2}\left(p_{1}\right)\right]^{2}\right\}} .
$$

In the analysis that follows we will use both the $\mathrm{BC}$ and the $\mathrm{CP}$ vertices, and compare the difference they induce to the various quantities of interest.

\section{B. The quark loop}

Let us now turn to the quark-loop diagram $\left(a_{11}\right)$ of the PT-BFM scheme. Factoring out the trivial color structure $\delta^{a b}$, we obtain

$$
\widehat{X}^{\mu \nu}\left(q^{2}\right)=-g^{2} d_{f} \int_{k} \operatorname{Tr}\left[\gamma^{\mu} S(k) \widehat{\Gamma}^{\nu}(k+q,-k,-q) S(k+q)\right],
$$

where $d_{f}$ is the Dynkin index of the fundamental representation $\left[d_{f}=1 / 2\right.$ for $\left.S U(3)\right]$.

Since by virtue of the WI (3.4) the quark loop $\widehat{X}^{\mu \nu}\left(q^{2}\right)$ is transverse ${ }^{1}$,

$$
q_{\mu} \widehat{X}^{\mu \nu}\left(q^{2}\right)=0
$$

\footnotetext{
${ }^{1}$ Note that the corresponding quark-loop in the covariant gauges, i.e., with $\widehat{\Gamma}^{\nu} \rightarrow \Gamma^{\nu}$ is also transverse, by virtue of the STI (3.3).
} 
we have that $\widehat{X}^{\mu \nu}\left(q^{2}\right)=\widehat{X}\left(q^{2}\right) P^{\mu \nu}(q)$; then, contracting with $g_{\mu \nu}$, and setting $d_{f}=1 / 2$, we obtain

$$
\widehat{X}\left(q^{2}\right)=-\frac{g^{2}}{2(d-1)} \int_{k} \operatorname{Tr}\left[\gamma^{\mu} S(k) \widehat{\Gamma}_{\mu}(k+q,-k,-q) S(k+q)\right] .
$$

After inserting the full vertex (3.5) into (3.13) and taking the trace, we find one term for each of the form factors $L_{i}$. Specifically, we have that

$$
\widehat{X}\left(q^{2}\right)=-\frac{2 g^{2}}{d-1} \int_{k} \frac{1}{A_{a} A_{b}\left(k^{2}-\mathcal{M}_{a}^{2}\right)\left[(k+q)^{2}-\mathcal{M}_{b}^{2}\right]} \sum_{i=1}^{3} T_{i}(k, k+q),
$$

where the subindex " $a$ " (respectively, " $b$ ") indicates that the corresponding function is evaluated at momentum $k$ (respectively, $k+q$ ), with

$$
\begin{aligned}
T_{1}(k, k+q) & =L_{1}\left\{(2-d)\left(k^{2}+k \cdot q\right)+d \mathcal{M}_{a} \mathcal{M}_{b}\right\} \\
T_{2}(k, k+q) & =L_{2}\left\{2[k \cdot(2 k+q)][(k+q) \cdot(2 k+q)]-k \cdot(k+q)(2 k+q)^{2}\right. \\
& \left.+(2 k+q)^{2} \mathcal{M}_{a} \mathcal{M}_{b}\right\}, \\
T_{3}(k, k+q) & =L_{3}\left\{\mathcal{M}_{b}[(2 k+q) \cdot k]+\mathcal{M}_{a}[(2 k+q) \cdot(k+q)]\right\} .
\end{aligned}
$$

Before studying in detail each term, let us consider $\widehat{X}\left(q^{2}\right)$ in the limit $q \rightarrow 0$. Using the expressions given in Eq. (3.6), and dropping the subindices (all quantities being evaluated at $k$ now), one finds

$$
\widehat{X}(0)=-\frac{2 g^{2}}{d-1} \int_{k} \frac{1}{A^{2}\left(k^{2}-\mathcal{M}^{2}\right)^{2}}\left\{A\left[(2-d) k^{2}+d \mathcal{M}^{2}\right]+2 A^{\prime} k^{2}\left(k^{2}+\mathcal{M}^{2}\right)-4 k^{2} B^{\prime} \mathcal{M}\right\} .
$$

The important point to recognize now is that the integral on the rhs of (3.16) vanishes by virtue of an identity valid in dimensional regularization. This identity, referred to as the "seagull identity" in the recent literature [54] constitutes the generalization of the simple identity (A3) employed in the Appendix for the one-loop perturbative result.

Specifically, the seagull identity reads

$$
\int_{k} k^{2} f^{\prime}\left(k^{2}\right)+\frac{d}{2} \int_{k} f\left(k^{2}\right)=0
$$

where the "prime" denotes differentiation with respect to $k^{2}$, i.e., $f^{\prime}\left(k^{2}\right) \equiv \frac{\mathrm{d} f\left(k^{2}\right)}{\mathrm{d} k^{2}}$. Interestingly enough, using inside Eq. (3.17) the function

$$
f\left(k^{2}\right)=\left[A\left(k^{2}\right)\left(k^{2}-\mathcal{M}^{2}\left(k^{2}\right)\right)\right]^{-1},
$$


namely the all order generalization of the one-loop $\left(k^{2}-M^{2}\right)^{-1}$ employed in Eq. (A3), one obtains precisely the integral on the rhs of (3.16); therefore,

$$
\widehat{X}(0)=0
$$

as announced.

Let us now compute the quark self-energy $\widehat{X}^{\mathrm{CP}}\left(q^{2}\right)$ obtained by substituting into (3.11) the vertex $\widehat{\Gamma}_{\mathrm{CP}}^{\mu}$, given in (3.9)$-(\underline{3.10})$. The answer will be expressed as a deviation from $\widehat{X}\left(q^{2}\right)$, namely

$$
\widehat{X}^{C P}\left(q^{2}\right)=\widehat{X}\left(q^{2}\right)+\delta \widehat{X}\left(q^{2}\right)
$$

where

$$
\delta \widehat{X}\left(q^{2}\right)=2 g^{2} \int_{k} \frac{\left[\mathcal{M}_{a} \mathcal{M}_{b}-\left(k^{2}+k \cdot q\right)\right]\left[(k+q)^{2}-k^{2}\right]}{A_{a} A_{b}\left(k^{2}-\mathcal{M}_{a}^{2}\right)\left[(k+q)^{2}-\mathcal{M}_{b}^{2}\right]} \widehat{\Gamma}_{\mathrm{T}}(k+q,-k,-q),
$$

It is easy to verify that the integral on the rhs of (3.21) vanishes in the limit $q \rightarrow 0$, because, as can be seen directly from (3.10), $\widehat{\Gamma}_{\mathrm{T}}\left(p_{1},-p_{1}, 0\right)=0$. Therefore, the property of (3.16) persists, namely $\widehat{X}^{\mathrm{CP}}(0)=0$.

\section{Renormalization}

Clearly $\widehat{X}\left(q^{2}\right)$ (and $\widehat{X}^{\mathrm{CP}}\left(q^{2}\right)$ ) must be renormalized within the momentum subtraction (MOM) scheme. This choice is dictated by the fact that our final results will be expressed as deviations from the quenched gluon propagator obtained from the lattice, where the latter scheme has been employed. The renormalized expression for $\widehat{X}\left(q^{2}\right)$ in the MOM scheme is given by

$$
\widehat{X}_{R}\left(q^{2}\right)=\widehat{X}\left(q^{2}\right)-\frac{q^{2}}{\mu^{2}} \widehat{X}\left(\mu^{2}\right)
$$

As far as the propagator of (2.20) is concerned, its renormalization will proceed as follows. First of all, as happens almost exclusively at the level of SDEs, the renormalization must be carried out subtractively instead of multiplicatively. The main reason for that is the mishandling of overlapping divergences due to the ambiguity inherent in the gauge-

technique construction of the vertex, related with the unspecified transverse part [48]. The (subtractive) renormalization must be carried out at the level of (2.13). Specifically,

$$
\Delta_{Q, R}^{-1}\left(q^{2}\right)=\frac{Z_{A} q^{2}+i\left[\widehat{\Pi}_{Q}\left(q^{2}\right)+\widehat{X}\left(q^{2}\right)\right]}{\left[1+G_{Q}\left(q^{2}\right)\right]^{2}}
$$


where the renormalization constant $Z_{A}$ is fixed in the MOM scheme through the condition $\Delta_{Q, R}^{-1}\left(\mu^{2}\right)=\mu^{2}$. This condition, when applied at the level of Eq. (3.23), allows one to express $Z_{A}$ as

$$
Z_{A}=\left[1+G_{Q}\left(\mu^{2}\right)\right]^{2}-\frac{i}{\mu^{2}}\left[\widehat{\Pi}_{Q}\left(\mu^{2}\right)+\widehat{X}\left(\mu^{2}\right)\right] .
$$

Now, as is well-known [50, 51], the validity of the BRST-driven relation (2.7) before and after renormalization prevents $G\left(\mu^{2}\right)$ from vanishing when, according to the MOM prescription, $F\left(\mu^{2}\right)=1$; instead, we must impose that $G\left(\mu^{2}\right)=-L\left(\mu^{2}\right)$. However, given that $L(x)$ is considerably smaller than $G(x)$ in the entire range of momenta, we can use the approximation $1+G\left(\mu^{2}\right) \approx F^{-1}\left(\mu^{2}\right)=1$, without introducing an appreciable numerical error [50, 51]. Thus, we obtain the following approximate equation for $Z_{A}$

$$
Z_{A}=1-\frac{i}{\mu^{2}}\left[\widehat{\Pi}_{Q}\left(\mu^{2}\right)+\widehat{X}\left(\mu^{2}\right)\right],
$$

Substituting Eq. (3.25) into Eq. (3.23), we obtain

$$
\Delta_{Q, R}^{-1}\left(q^{2}\right)=\frac{q^{2}+i\left[\widehat{\Pi}_{Q, R}\left(q^{2}\right)+\widehat{X}_{R}\left(q^{2}\right)\right]}{\left[1+G_{Q, R}\left(q^{2}\right)\right]^{2}},
$$

where, as in (3.22), $\widehat{\Pi}_{Q, R}\left(q^{2}\right)=\widehat{\Pi}_{Q}\left(q^{2}\right)-\frac{q^{2}}{\mu^{2}} \widehat{\Pi}_{Q}\left(\mu^{2}\right)$, while $G_{Q, R}\left(q^{2}\right)=G_{Q}\left(q^{2}\right)-G_{Q}\left(\mu^{2}\right)$.

On the other hand, the exact same procedure yields for the renormalized quenched propagator (setting $\widehat{X}=0$ and dropping the subscript "Q")

$$
\Delta_{R}^{-1}\left(q^{2}\right)=\frac{q^{2}+i \widehat{\Pi}_{R}\left(q^{2}\right)}{\left[1+G_{R}\left(q^{2}\right)\right]^{2}} .
$$

Then, according to the key operating assumption explained in the previous section, the unquenched quantities $\widehat{\Pi}_{Q}\left(q^{2}\right)$ and $G_{Q}\left(q^{2}\right)$ are to be approximated simply by their quenched counterparts, $\widehat{\Pi}\left(q^{2}\right)$ and $G\left(q^{2}\right)$, respectively. Consequently, it is easy to verify that the renormalized version of (2.20) is given by

$$
\Delta_{Q, R}\left(q^{2}\right)=\frac{\Delta_{R}\left(q^{2}\right)}{1+\left\{i \widehat{X}_{R}\left(q^{2}\right)\left[1+G_{R}\left(q^{2}\right)\right]^{-2}-\lambda^{2}\right\} \Delta_{R}\left(q^{2}\right)} .
$$

In this context, the gluon mass related term $\lambda^{2}$ merits some additional comments. As has been emphasized amply in recent works, the seagull identity of (3.17), when applied to the gluon mass equation, enforces the annihilation of all quadratic divergences [53, 54]. This is a point of central importance, because the disposal of such divergences (had they 
survived) would require the introduction in the original Yang-Mills Lagrangian of a counterterm of the form $m_{0}^{2} A_{\mu}^{2}$, which is, however, forbidden by the local gauge invariance, which must remain intact. Therefore, at least in principle, the renormalization of the gluon mass equation proceeds as in the case of the homogeneous quark mass equation (obtained from the corresponding gap equation without a current mass term), simply by renormalizing (multiplicatively) the various quantities appearing on its rhs [53]. Note, however, that these considerations, theoretically important as they may be, are of limited practical relevance for the present work, because, as already mentioned, the quantity $\lambda^{2}$ will be not determined dynamically, but rather fitted from the (extrapolated) solutions obtained.

\section{The transition to Euclidean space}

The actual calculations will be carried out in the Euclidean space, and the various relevant formulas, most notably (2.20) and (3.28), must be modified accordingly. In particular, the integral measure is given by

$$
\int_{k}=i \int_{k_{\mathrm{E}}}=\frac{i}{(2 \pi)^{d}} \frac{\pi^{\frac{d-1}{2}}}{\Gamma\left(\frac{d-1}{2}\right)} \int_{0}^{\pi} \mathrm{d} \theta \sin ^{d-2} \theta \int_{0}^{\infty} \mathrm{d} y y^{\frac{d}{2}-1},
$$

where $y=k^{2}$. When $d=4$ this reduces to

$$
\int_{k}=\frac{i}{(2 \pi)^{3}} \int_{0}^{\pi} \mathrm{d} \theta \sin ^{2} \theta \int_{0}^{\infty} \mathrm{d} y y=\frac{i}{(2 \pi)^{3}} \int_{\mathrm{E}}
$$

which is the measure employed in our final results. In addition, we will use the standard formulas that allow the transition of the various Green's functions from the physical Minkowski momentum $q^{2}$ to the Euclidean $q_{\mathrm{E}}^{2}=-q^{2}>0$; specifically

$$
\Delta_{\mathrm{E}}\left(q_{\mathrm{E}}^{2}\right)=-\Delta\left(-q_{\mathrm{E}}^{2}\right) ; \quad F_{\mathrm{E}}\left(q_{\mathrm{E}}^{2}\right)=F\left(-q_{\mathrm{E}}^{2}\right) ; \quad G_{\mathrm{E}}\left(q_{\mathrm{E}}^{2}\right)=G\left(-q_{\mathrm{E}}^{2}\right)
$$

and

$$
A_{\mathrm{E}}\left(q_{\mathrm{E}}^{2}\right)=A\left(-q_{\mathrm{E}}^{2}\right) \quad B_{\mathrm{E}}\left(q_{\mathrm{E}}^{2}\right)=B\left(-q_{\mathrm{E}}^{2}\right) .
$$

The Euclidean version of $\widehat{X}\left(q^{2}\right)$ is defined as the result of the aforementioned operations at the level of (3.14), but with the imaginary factor $i$ that comes from the measure absorbed by the factor of $i$ multiplying $\widehat{X}\left(q^{2}\right)$ in Eqs. (2.20) or (3.28). Effectively, this amounts to the substitution $i \widehat{X}\left(q^{2}\right) \rightarrow-\widehat{X}_{\mathrm{E}}\left(q_{\mathrm{E}}^{2}\right)$ where the $\widehat{X}_{\mathrm{E}}\left(q_{\mathrm{E}}^{2}\right)$ is obtained from (3.14) by replacing 
$\int_{k} \rightarrow \int_{k_{\mathrm{E}}}$, (no more $i$ ) euclidianizing the momenta $\left(q^{2} \rightarrow-q_{\mathrm{E}}^{2}, k^{2} \rightarrow-k_{\mathrm{E}}^{2}\right)$, and using (3.32). Then, the euclidian version of (2.20) becomes (we suppress the suffix "E" throughout)

$$
\Delta_{Q}\left(q^{2}\right)=\frac{\Delta\left(q^{2}\right)}{1+\left\{\widehat{X}\left(q^{2}\right)\left[1+G\left(q^{2}\right)\right]^{-2}+\lambda^{2}\right\} \Delta\left(q^{2}\right)} .
$$

The conversion of (3.28) to Euclidean space proceeds following exactly analogous steps.

One may carry out two elementary checks of the expression given in (3.33). First, in the IR limit, $q^{2}=0$, after using (3.16), $\Delta^{-1}(0)=m^{2}(0)$, and the definition of $\lambda^{2}$ in (2.18), we obtain $\Delta_{Q}^{-1}(0)=m_{Q}^{2}(0)$, as we should.

In the opposite limit, where $q^{2}$ acquires large values compared to all mass scales involved, we substitute into Eq. (3.33) the perturbative one-loop results, keeping terms up to order $\alpha_{s}$. The Euclidean version of (A11) is determined following the steps described above; specifically, since

$$
i \widehat{X}^{[1]}\left(q^{2}\right)=-\frac{\alpha_{s}}{6 \pi} q^{2} \ln \left(-q^{2} / \mu^{2}\right),
$$

then (restoring the "E" for this step only)

$$
\begin{aligned}
\widehat{X}_{\mathrm{E}}^{[1]}\left(q_{\mathrm{E}}^{2}\right) & =\left\{\frac{\alpha_{s}}{6 \pi} q^{2} \ln \left(-q^{2} / \mu^{2}\right)\right\}_{q^{2} \rightarrow-q_{\mathrm{E}}^{2}} \\
& =-\frac{\alpha_{s}}{6 \pi} q_{\mathrm{E}}^{2} \ln \left(q_{\mathrm{E}}^{2} / \mu^{2}\right) .
\end{aligned}
$$

Combining this with the standard result

$$
\left[\Delta^{-1}\left(q^{2}\right)\right]^{[1]}=q^{2}\left[1+\frac{13 C_{A} \alpha_{s}}{24 \pi} \ln \left(q^{2} / \mu^{2}\right)\right]
$$

we obtain from (3.33) [with $n_{f}$ quark flavors, and $C_{A}=3$ ]

$$
\left[\Delta_{Q}^{-1}\left(q^{2}\right)\right]^{[1]}=q^{2}\left[1+\frac{\alpha_{s}}{8 \pi}\left\{13-\frac{4}{3} n_{f}\right\} \ln \left(q^{2} / \mu^{2}\right)\right],
$$

which is the correct one-loop result (in the Landau gauge).

In the derivation given above, the perturbative expression for $G$, namely $\left(C_{A}=3\right)$

$$
1+G^{[1]}\left(q^{2}\right)=1+\frac{9 \alpha_{s}}{16 \pi} \ln \left(q^{2} / \mu^{2}\right)
$$

was not necessary, since, its inclusion in Eq. (3.33) gives contributions of $\mathcal{O}\left(g^{4}\right)$; however, (3.38) is needed for a final check. Specifically, as is well known, due to the QED-like WIs characteristic of PT-BFM scheme, the PT-BFM propagator, usually denoted by $\widehat{\Delta}$, captures 
the running of the gauge coupling ( $\beta$ function), for any value of the gauge-fixing parameter. $\widehat{\Delta}$ and $\Delta$ are related by the all-order relation $[42,60]$

$$
\widehat{\Delta}^{-1}\left(q^{2}\right)=\left[1+G\left(q^{2}\right)\right]^{2} \Delta^{-1}\left(q^{2}\right)
$$

whose perturbative expansion yields

$$
\left[\widehat{\Delta}_{Q}^{-1}\left(q^{2}\right)\right]^{[1]}=q^{2}\left[1+\frac{\alpha_{s}}{48 \pi}\left\{33-2 n_{f}\right\} \ln \left(q^{2} / \mu^{2}\right)\right]
$$

namely the correct one-loop result.

\section{NUMERICAL RESULTS}

In this section we will first review the lattice data for the quenched gluon propagator $\Delta\left(q^{2}\right)$ and ghost dressing function $F\left(q^{2}\right)$, and the nonperturbative expressions of the quark functions $A\left(p^{2}\right)$ and $B\left(p^{2}\right)$, obtained in [31] from the solution of the quark gap equation. With all necessary ingredients available, i.e., $\Delta\left(q^{2}\right), F\left(q^{2}\right), A\left(q^{2}\right)$ and $B\left(q^{2}\right)$, we then evaluate numerically the integrals that determine the contribution of the quark loop, $\widehat{X}\left(q^{2}\right)(\mathrm{BC}$ vertex), and $\widehat{X}^{\mathrm{CP}}\left(q^{2}\right)$ (CP vertex), given by Eqs. (3.14) and (3.20), respectively. Finally, with the quark loop contribution at our disposal, we proceed to estimate through Eq. (3.33) the effect of "unquenching", namely how the overall shape of the quenched propagator $\Delta\left(q^{2}\right)$ is affected by the presence of the quark loops. Finally, we compare the resulting dressing function with that obtained from unquenched lattice simulations. Given the amount of information presented in this section, we have organized the material in four subsections, and have enumerated the main points of each subsection, to facilitate the perusal.

\section{A. Ingredients}

(i) The starting point of our numerical analysis are the quenched $S U(3)$ lattice results for the gluon propagator $\Delta\left(q^{2}\right)$ and ghost dressing function $F\left(q^{2}\right)$ [7]. These are shown, respectively, on the left and right panels of Fig. 6, for three different renormalization points $(\mu=4.3,3.0$ and $2.3 \mathrm{GeV})$. On the same figure we also plot the corresponding fits for the three different renormalization points; the explicit functional form used for these fits can be found in various recent articles [30, 31, 53]. 

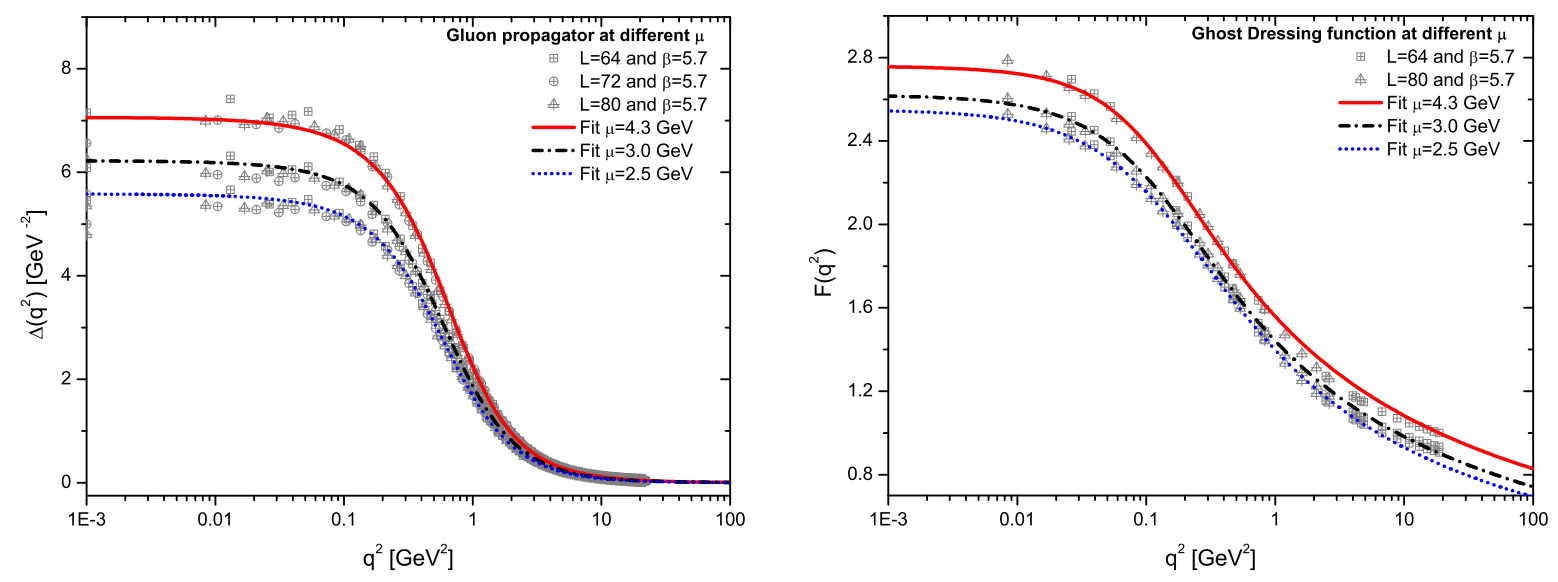

FIG. 6: Lattice result for the $S U(3)$ gluon propagator (left panel) and ghost dressing function (right panel) renormalized at three different points: $\mu=4.3 \mathrm{GeV}$ (solid red curve), $\mu=3.0 \mathrm{GeV}$ (dash-dotted black curve), and $\mu=2.5 \mathrm{GeV}$ (dotted blue curve).

(ii) Next, the computation of the quark contribution $\widehat{X}\left(q^{2}\right)$ and $\widehat{X}^{\mathrm{CP}}\left(q^{2}\right)$ [Eqs. (3.14) and (3.20)] requires the knowledge of the nonperturbative behavior of the functions $A\left(k^{2}\right)$ and $B\left(k^{2}\right)$ appearing in the definition of the full quark propagator (3.7). Both functions can be determined by solving numerically the quark gap equation; however, one has to be particular careful on how the non-Abelian quark-gluon vertex, which enters in the latter equation, is approximated. Note in particular that, as discussed in detail in [31], the quark gap equation is identical within both the conventional and the PT-BFM frameworks. As a result, the quark-gluon vertex entering in it is $\Gamma_{\mu}$ (and not $\widehat{\Gamma}_{\mu}$ ), satisfying the STI given in Eq. (3.3). This fact, in turn, introduces a numerically crucial dependence on the ghost dressing function and the quark-ghost scattering amplitude. Once these effects are duly taken into account, and the $\mathrm{BC}$ or $\mathrm{CP}$ vertices improved accordingly [31], one can solve the resulting nonlinear system of integral equations for $A\left(k^{2}\right)$ and $B\left(k^{2}\right)$, supplemented by the lattice gluon propagator and ghost dressing function mentioned above.

(iii) The results obtained following the outlined procedure are shown in Fig. 7 (for the specific value $\mu=4.3 \mathrm{GeV}$ in this case). In particular, on the left panel we plot the inverse of the quark wave function $A^{-1}\left(k^{2}\right)$ for the improved BC vertex (dotted black curve), and the "improved" CP vertex (dashed blue curve); on the right panel we show the corresponding solutions for the $B\left(k^{2}\right)$ function. At this point the momentum dependence of the dynamical quark mass $\mathcal{M}\left(k^{2}\right)$ can be straightforwardly obtained, since $\mathcal{M}\left(k^{2}\right)=B\left(k^{2}\right) / A\left(k^{2}\right)$, and 

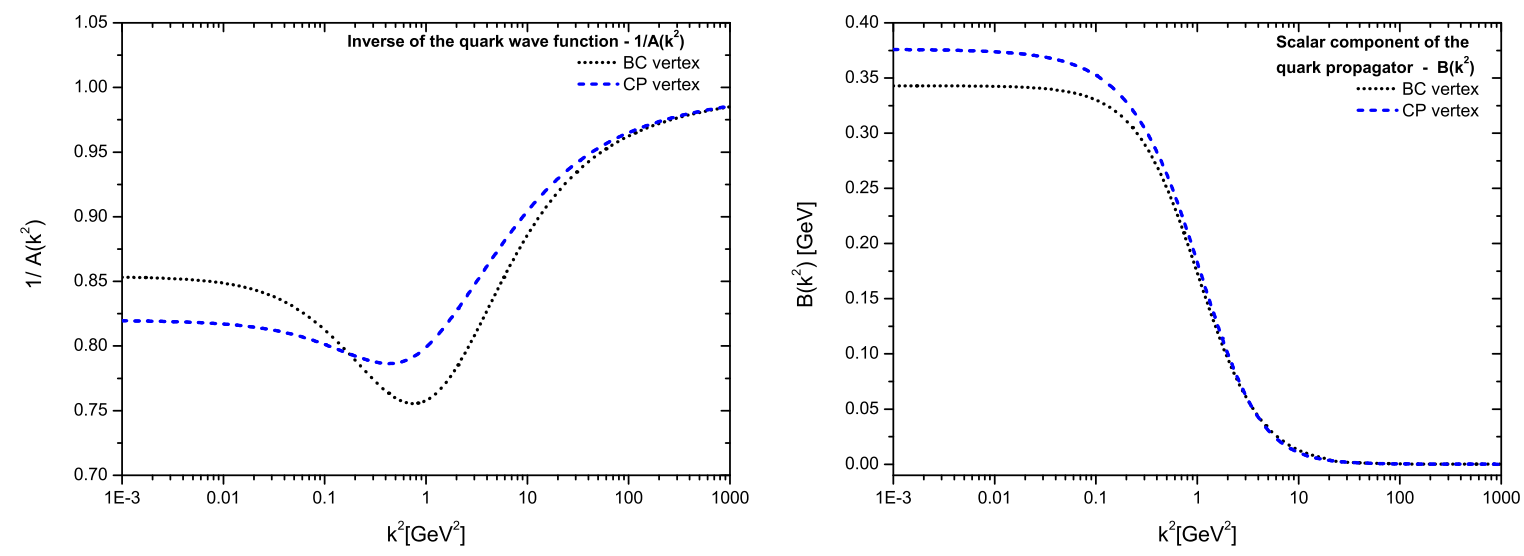

FIG. 7: Solution of the quark gap equation: $A^{-1}\left(k^{2}\right)$ (left panel) and $B\left(k^{2}\right)$ (right panel) renormalized at $\mu=4.3 \mathrm{GeV}$. Dotted black curves correspond to the improved BC vertex, while dashed blue curves to the improved $\mathrm{CP}$ vertex.

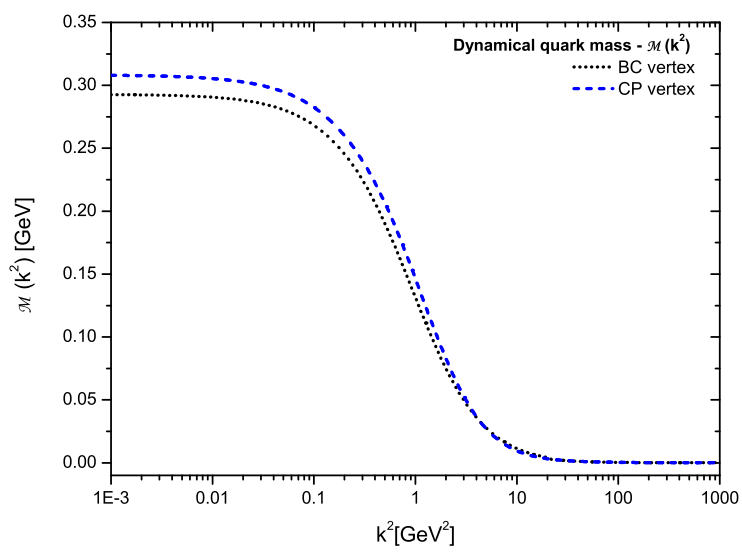

FIG. 8: The momentum dependence of the dynamical quark mass $\mathcal{M}\left(k^{2}\right)=B\left(k^{2}\right) / A\left(k^{2}\right)$, using the same conventions as in the previous plot.

is plotted in Fig. 8, for the two forms of the quark gluon vertex considered. Clearly the two results coincide in the $\mathrm{UV}$, whereas in the IR we notice that the $\mathrm{CP}$ vertex produces the slightly higher value $\mathcal{M}(0)=M=307 \mathrm{MeV}$ when compared with the $\mathrm{BC}$ vertex result $M=292 \mathrm{MeV}$. Note, finally, that the results presented have been obtained in the chiral limit, where no "current" mass has been used when solving the gap equation. 

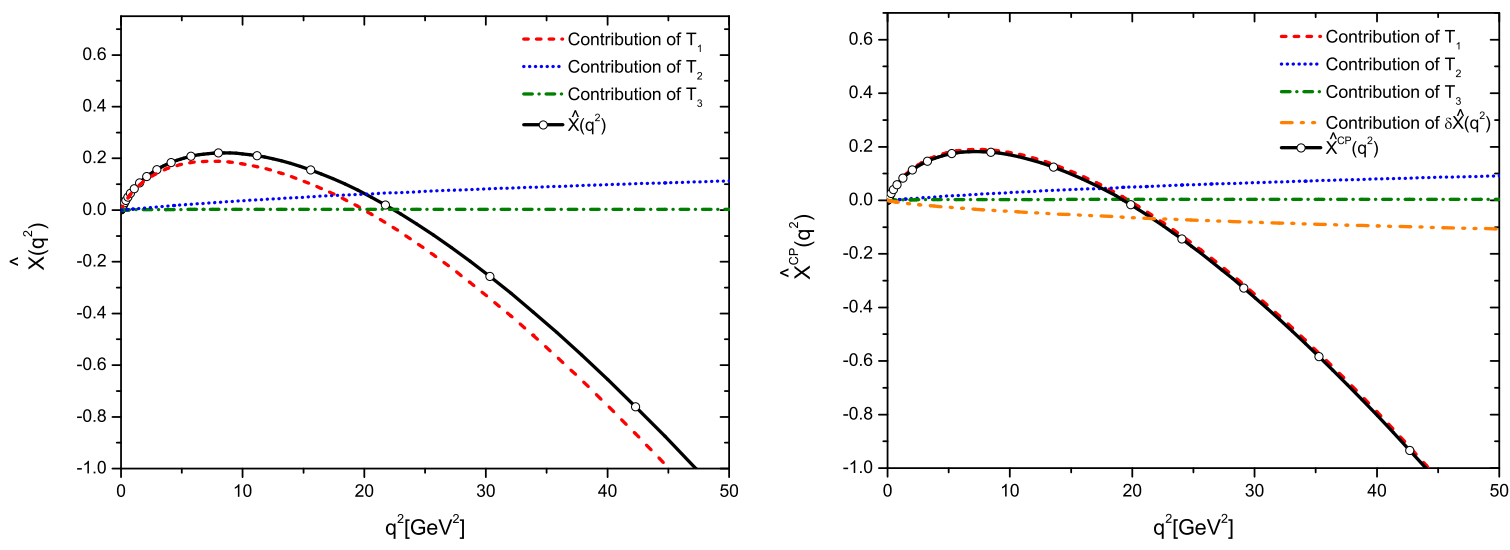

FIG. 9: Individual contributions of the terms proportional to $T_{1}$ (dashed red curve), $T_{2}$ (dotted blue line), $T_{3}$ (dash-dotted green) and $\delta \widehat{X}\left(q^{2}\right)$ (dashed with two dots orange curve), to $\widehat{X}\left(q^{2}\right)$ (left panel) and $\widehat{X}^{\mathrm{CP}}\left(q^{2}\right)$ (right panel) respectively. The sum of all contributions produce in both cases the continuous (black with white circles) curve, which represent the full quark loop contribution to the gluon propagator.

\section{B. The quark loop}

We can now proceed to the numerical evaluation of the full quark loop, namely $\widehat{X}\left(q^{2}\right)$ (BC vertex) and $\widehat{X}^{\mathrm{CP}}\left(q^{2}\right)$ (CP vertex), as given by Eqs. (3.14) and (3.20) respectively.

( $i$ ) On the left panel of Fig. 9 we show the results obtained for each individual contribution of $\widehat{X}\left(q^{2}\right)$, as expressed in Eqs. (3.14) and (3.15). As can be easily seen, the leading contribution comes from the $T_{1}$ term, which, as shown in the Appendix, is also the term responsible for the appearance of the perturbative logarithm; the $T_{2}$ and $T_{3}$ contributions are instead subdominant.

(ii) On the right panel of Fig. 9, we show the same quantities for the $\widehat{X}^{\mathrm{CP}}\left(q^{2}\right)$ term. In this case, one has the additional contribution $\delta \widehat{X}\left(q^{2}\right)$, given in Eq. (3.21), coming from the inclusion of the transverse part of the quark-gluon vertex. The net numerical effect is that the latter term will almost completely cancel the subdominant terms $T_{2}$ and $T_{3}$, so that the $T_{1}$ term practically coincides with the full answer.

(iii) The results for the quark loop $\widehat{X}\left(q^{2}\right)$ and $\widehat{X}^{\mathrm{CP}}\left(q^{2}\right)$ are finally compared in Fig. 10 for the $n_{f}=2$ case. It is important to notice that, indeed, $\widehat{X}(0)=\widehat{X}^{\mathrm{CP}}(0)=0$, as we had previously announced. 


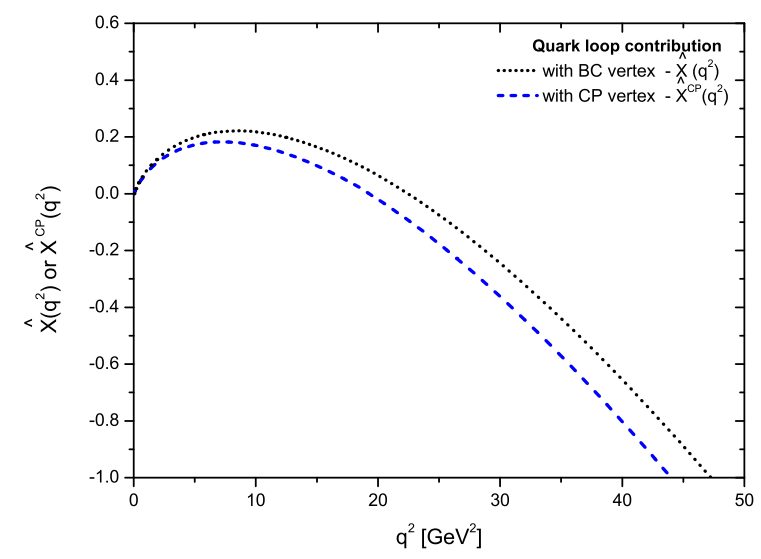

FIG. 10: Comparison between the contributions of the quark loop $\widehat{X}\left(q^{2}\right)$ (dotted black curve) and $\widehat{X}^{\mathrm{CP}}\left(q^{2}\right)$ (dashed blue curve) to the gluon self-energy with $n_{f}=2$.

\section{Effect on the gluon propagator}

(i) The next step is to compute the unquenched gluon propagator given in Eq. (3.33). The first thing we should notice is the presence of the auxiliary function $1+G\left(q^{2}\right)$ in the denominator of Eq. (3.33). Using the fact that, in the Landau gauge, $L\left(q^{2}\right)$ is numerically suppressed [50, 51], it follows immediately from Eq. (2.7) that $1+G\left(q^{2}\right) \approx F^{-1}\left(q^{2}\right)$.

(ii) Substituting into Eq. (3.33) the results for $\Delta\left(q^{2}\right)$ and $F\left(q^{2}\right)$, renormalized at $\mu=4.3 \mathrm{GeV}$ and presented in Fig. 6, together with either $\widehat{X}\left(q^{2}\right)$ (BC vertex) or $\widehat{X}^{\mathrm{CP}}\left(q^{2}\right)$ (CP vertex) of Fig. 10, we obtain the results shown on the left panel of Fig. 11. As before, the dotted black curve represents the result for the case where we employ the BC vertex, while the dashed blue curve is for the $\mathrm{CP}$ vertex. We clearly see that the unquenched gluon propagator suffers a sizable suppression in the intermediate momenta region compared to the quenched case (solid red curve). Notice that, in this particular case (left panel of Fig. 11) we have set $\lambda^{2}=0$, and therefore, the three curves coincides at $q^{2}=0$ since $\widehat{X}(0)=\widehat{X}^{\mathrm{CP}}(0)=0$.

(iii) As mentioned before, due to our present limitation in determining the precise value of $\lambda^{2}$, we will restrict ourselves to extracting an approximate range for $\lambda^{2}$, through the extrapolation of the curves in the region delimited by the shaded area showed on the left panel of Fig. 11,

Specifically, we perform a one-dimensional extrapolation in the deep IR region using as input the result obtained for the quenched gluon propagator in the middle IR and intermediate regions. The first step is to select the momentum from which $\Delta_{Q}(q)$ is extrapolated. 

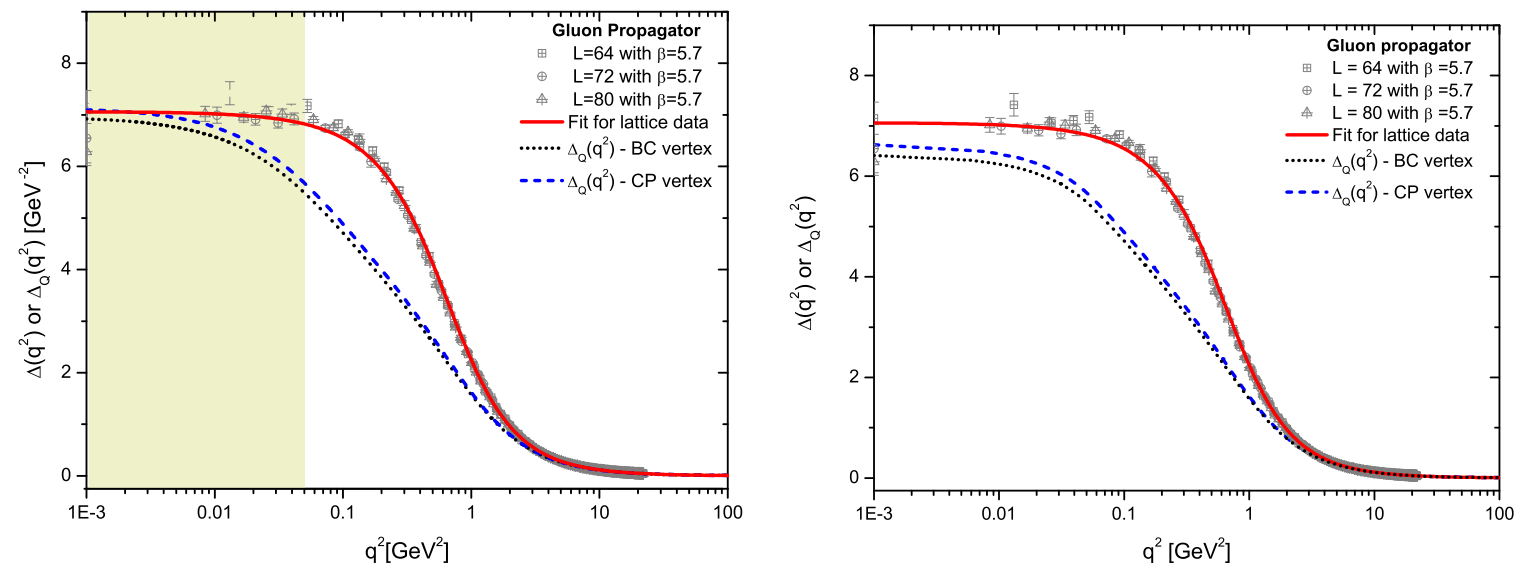

FIG. 11: The unquenched gluon propagator $\Delta_{Q}\left(q^{2}\right)$ when no extrapolation is used, i.e. $\lambda^{2}=0$ in Eq. (3.33) (left panel). The dotted black curve represents the unquenched propagator obtained with the BC vertex whereas the dashed blue curve represents the result for the $\mathrm{CP}$ vertex. The result for $\Delta_{Q}\left(q^{2}\right)$ when the extrapolation is performed in the shade area i.e. from $q^{2}=0.05 \mathrm{GeV}^{2}$ towards the deep IR (right panel).

We choose three different points, namely (i) $0.02 \mathrm{GeV}^{2}$, (ii) $0.05 \mathrm{GeV}^{2}$ and (iii) $0.07 \mathrm{GeV}^{2}$, and implement the extrapolation starting for each of these points. In all three cases, we extrapolate the data up to $q^{2}=10^{-3} \mathrm{GeV}^{2}$ using the cubic B-spline method. We basically split each of these ranges into a 150 pieces, and fit each segment with a cubic Bezier spline. The goal is to get a fit segment that is smooth in the first derivative, and continuous in the second derivative, both within an interval and at its boundaries. When these boundary conditions are met, the entire function is constructed in a piece-wise manner.

On the right panel of Fig. 11, we show $\Delta_{Q}\left(q^{2}\right)$ when the extrapolation is done for values of momenta smaller than $q^{2}=0.05 \mathrm{GeV}^{2}$. As we can clearly see, the tendency of the unquenched gluon propagator is always to be below the quenched one (solid red curve), no matter if we use the BC vertex (dotted black curve) or the CP vertex (dashed blue curve).

Now, we are in position to determine the order of magnitude of $m_{Q}^{2}(0)$ and $\lambda^{2}$. Combining Eqs. (2.15) and (2.18) and the data presented on the right panel of Fig. 11, we found the values of $m_{Q}^{2}(0)=0.156 \mathrm{GeV}^{2}, m^{2}(0)=0.142 \mathrm{GeV}^{2}$ and $\lambda^{2}=0.014 \mathrm{GeV}^{2}$ for the $\mathrm{BC}$ vertex, whereas for the $\mathrm{CP}$ we have $m_{Q}^{2}(0)=0.151 \mathrm{GeV}^{2}$ and $\lambda^{2}=0.009 \mathrm{GeV}^{2}$. These results suggest that the effective gluon mass increases when we include the quark loops in the gluon self-energy. 


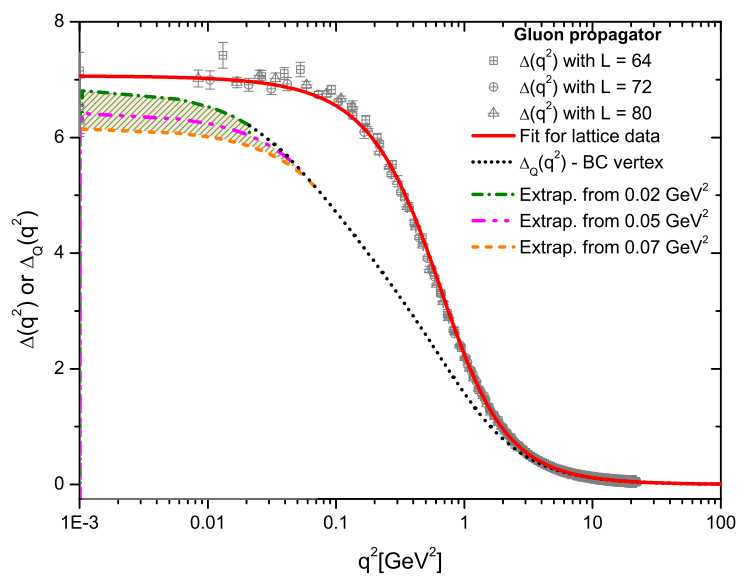

FIG. 12: Comparison between the quenched $\Delta\left(q^{2}\right)$ and the unquenched $\Delta_{Q}\left(q^{2}\right)$ gluon propagators. The yellow striped band shows the possible values that $\Delta_{Q}(0)$ can assume at zero momentum. The two curves delimiting the band represent the extrapolation towards the IR either starting from $0.02 \mathrm{GeV}^{2}$ (dash-dotted green line) or $0.07 \mathrm{GeV}^{2}$ (dashed orange line). The dashed with two dots magenta curve corresponds to an extrapolation of the numerical result starting from the intermediate value $0.05 \mathrm{GeV}^{2}$.

In addition, notice that the results obtained with the $\mathrm{BC}$ and $\mathrm{CP}$ vertices differ only by approximately $3 \%$. Since this difference is rather small and does not cause significant changes in what follows, for the rest of our analysis we will focus on the BC vertex only.

(iv) It is important to verify whether the IR suppression, shown in the unquenched propagator of the right panel of Fig. 11, persists when we start the curve extrapolation from different values. This is shown in Fig. 12, where we compare the results obtained when we extrapolate $\Delta_{Q}\left(q^{2}\right)$ (with the $\mathrm{BC}$ vertex) from momenta below $q^{2}=0.02 \mathrm{GeV}^{2}$ (dashdotted green line), $q^{2}=0.05 \mathrm{GeV}^{2}$ (dashed with two dots magenta line), and $q^{2}=0.07 \mathrm{GeV}^{2}$ (dashed orange line).

Indeed, we can see that the general trend, for all cases, is that the unquenched propagator $\Delta_{Q}\left(q^{2}\right)$ displays suppressed intermediate and IR regions, when compared to the quenched case. In particular, for the extrapolation starting at $q^{2}=0.02 \mathrm{GeV}^{2}$ we can see that $m_{Q}^{2}(0)=0.147 \mathrm{GeV}^{2}$ and $\lambda^{2}=0.005 \mathrm{GeV}^{2}$; whereas when we extrapolate from $q^{2}=0.07 \mathrm{GeV}^{2}$ we obtain $m_{Q}^{2}(0)=0.163 \mathrm{GeV}^{2}$ and $\lambda^{2}=0.021 \mathrm{GeV}^{2}$. Therefore, the extrapolations mentioned above produce a range of possible values for $\Delta_{Q}(0)$ or, equivalently, 

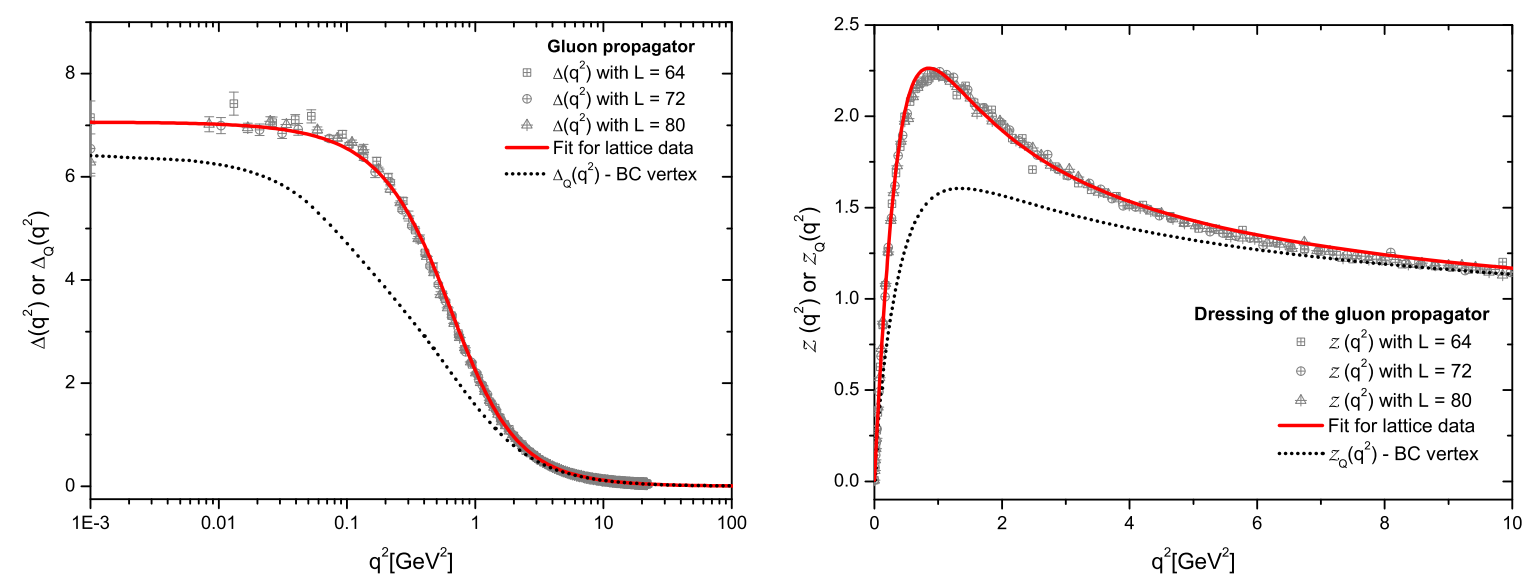

FIG. 13: The quenched (solid red curve) and unquenched (dotted black curve) gluon propagators (left panel) and dressing functions (right panel). The unquenched case corresponds to the case where the extrapolation starts at $q^{2}=0.05 \mathrm{GeV}^{2}$.

$m_{Q}^{2}(0)$ indicated by the yellow striped band on Fig. [12, where the difference between the upper and the lower value is approximately 10\%. Thus, the general conclusions we can draw with respect to the properties of the unquenched propagator are quite insensitive to the extrapolation point used (and therefore, ultimately, to the value of $\lambda$ ). In what follows we will further explore the properties of $\Delta_{Q}\left(q^{2}\right)$ extrapolated towards the IR starting from $q^{2}=0.05 \mathrm{GeV}^{2}$.

On the left panel of Fig. 13 we superimpose the quenched lattice result of [7] (solid red curve) and the unquenched result obtained from our calculation (dotted black curve), while on the right panel we show a comparison of the corresponding dressing functions. In the latter case notice that, as expected, both the quenched and unquenched curves vanish at zero momentum transfer, and their differences in the deep IR region is completely washed out. A direct comparison between the unquenched dressing function computed here and that obtained in the unquenched lattice simulation of [6] is postponed for the next subsection.

$(v)$ The dependence of the unquenched solution on the number of the flavors $n_{f}$ is next shown in Fig. 14, As in previous plots, we show the quenched lattice data (solid red curve) as a benchmark, while different dashed and/or dotted curves correspond to different values of flavors: $n_{f}=1$ (dash-dotted green curve), $n_{f}=2$ (dotted black curve), and, finally, $n_{f}=3$ (dashed blue curve). Evidently, increasing the number of flavors results in a more suppressed gluon propagator. As can be seen clearly in Fig. 14, in the IR and intermediate 


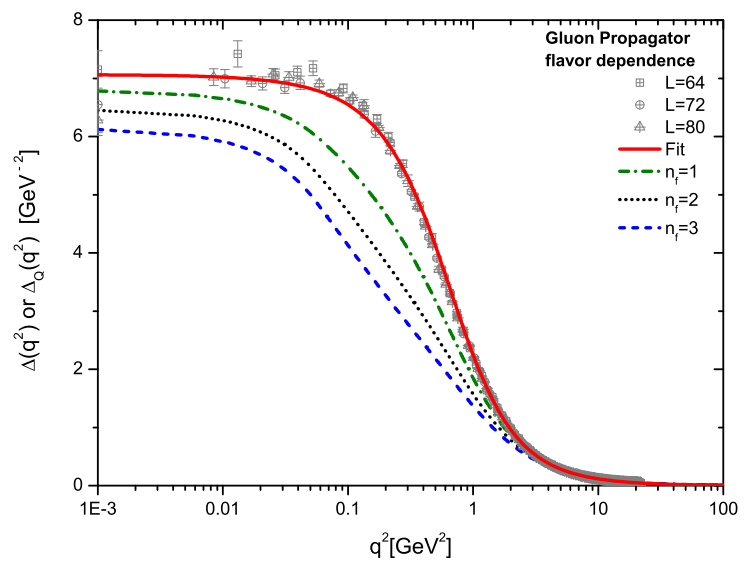

FIG. 14: The unquenched gluon propagator for different number of flavors: $n_{f}=1$ (dash-dotted green curve), $n_{f}=2$ (dotted black curve) and $n_{f}=3$ (dashed blue curve).

regions the curves with more active quarks lie below the ones with fewer. This fact does not contradict the one-loop perturbative behavior, given by Eq. (3.37), stating that, in the UV, $\Delta_{Q}\left(q^{2}\right)$ increases for a higher number of quark families. Indeed, we have checked that the perturbative behavior of $\Delta_{Q}\left(q^{2}\right)$ is recovered, due to a crossing that takes place around the renormalization point $\mu$, which makes the curve for $n_{f}=3$ (dashed blue curve) go above all the others in the perturbative regime.

(vi) In Fig. 15 we show another interesting property of $\Delta_{Q}\left(q^{2}\right)$. The dotted black curve represents $\Delta_{Q}\left(q^{2}\right)$ obtained with the nonperturbative expression for $\widehat{X}\left(q^{2}\right)$ given by Eqs. (3.14) and (3.15); the dash-dotted blue curve refers instead to the result of a simple one-loop calculation with a constant quark mass (see Eq. (A5) in the Appendix). Notice that the latter result can be obtained by substituting $A_{a}=A_{b}=1$ and $B_{a}=B_{b}=292 \mathrm{MeV}$ into Eqs. (3.14) and (3.15). The difference between the two curves is at the few percent level, in agreement with the observation made before that the terms $T_{2}$ and $T_{3}$ are numerically subdominant (at the one-loop level these terms vanish, since $L_{2}=L_{3}=0$ ). These observations suggest that the nonperturbative quark loop diagram $\left(a_{11}\right)$ appears to be rather insensitive to the running of the dynamical quark mass. 

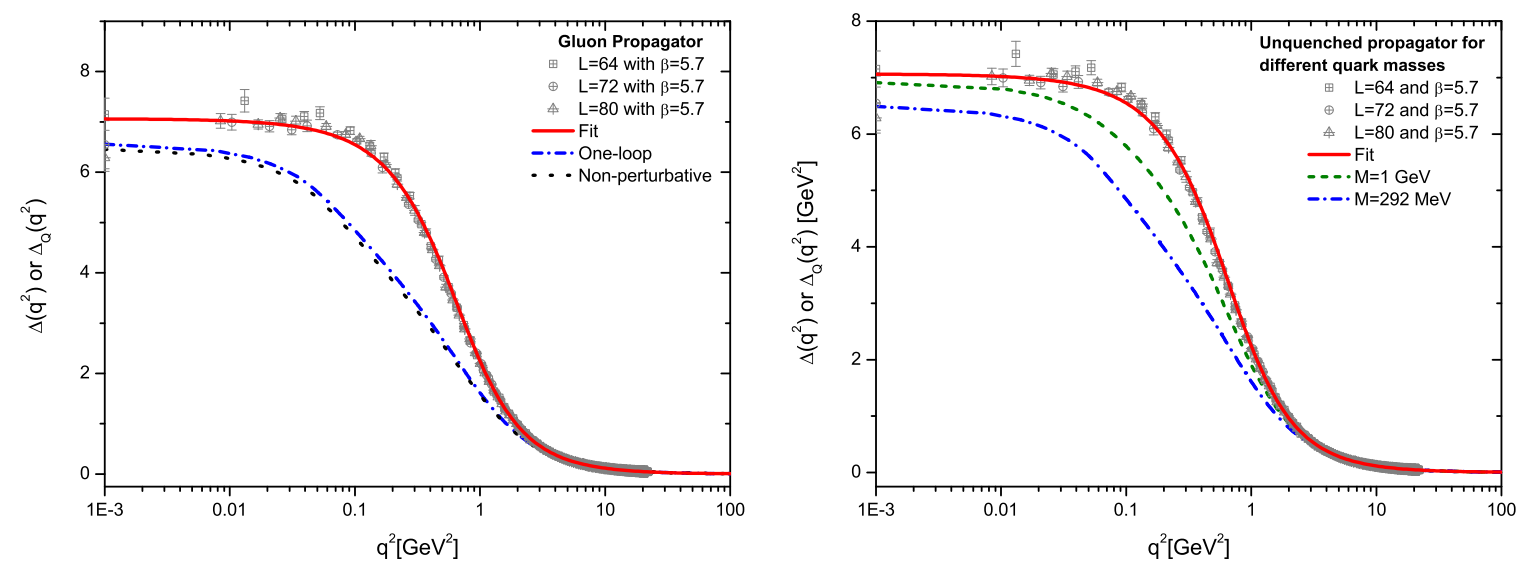

FIG. 15: Left panel: The unquenched gluon propagator $\Delta_{Q}\left(q^{2}\right)$ for $n_{f}=2$ (dotted black curve) compared to the one-loop dressed result with a constant quark mass $M=292 \mathrm{MeV}$ (dash-dotted blue curve). Right panel: The unquenched gluon propagator $\Delta_{Q}\left(q^{2}\right)$ for $n_{f}=2$ with different values of constant quark masses: $M=1 \mathrm{GeV}$ (dashed green curve) and $M=292 \mathrm{MeV}$ (dash-dotted blue curve).

(vii) To check the decoupling of the heavier flavors, we also compare in the right panel of the same figure the result of the one-loop calculation with $M=292 \mathrm{MeV}$ (dash-dotted blue curve) and $M=1 \mathrm{GeV}$ (dashed green curve). As we see clearly, the effect of the dynamical fermions on the gluon propagator becomes progressively suppressed as the quark mass increases.

(viii) Up to now, we have computed the unquenched gluon propagator $\Delta_{Q}\left(q^{2}\right)$ for a particular fixed value of the renormalization point $\mu$, namely $\mu=4.3 \mathrm{GeV}$. It is well-known that, both quenched and unquenched gluon propagators are $\mu$-dependent quantities, and therefore, different choices of $\mu$ will lead to different results.

In order to address quantitatively this effect, on the left panel of Fig. 16 we show $\Delta_{Q}\left(q^{2}\right)$ with $n_{f}=2$ for three different values of $\mu$ : (i) $\mu=2.5 \mathrm{GeV}$ and $\alpha_{s}=0.461$ (dotted blue curve); (ii) $\mu=3.0 \mathrm{GeV}$ and $\alpha_{s}=0.395$ (dash-dotted black curve), and (iii) $\mu=4.3 \mathrm{GeV}$ and $\alpha_{s}=0.295$ (solid red curve). Details on how the values of $\alpha_{s}$ corresponding to each renormalization point were determined can be found in [32, 51]. From Fig. 16] one can then clearly see that higher values of $\mu$ correspond to larger values of $\Delta_{Q}(0)$, which is basically the same pattern observed for the quenched case.

(ix) Finally, one important property that relates the gluon propagators renormalized at 

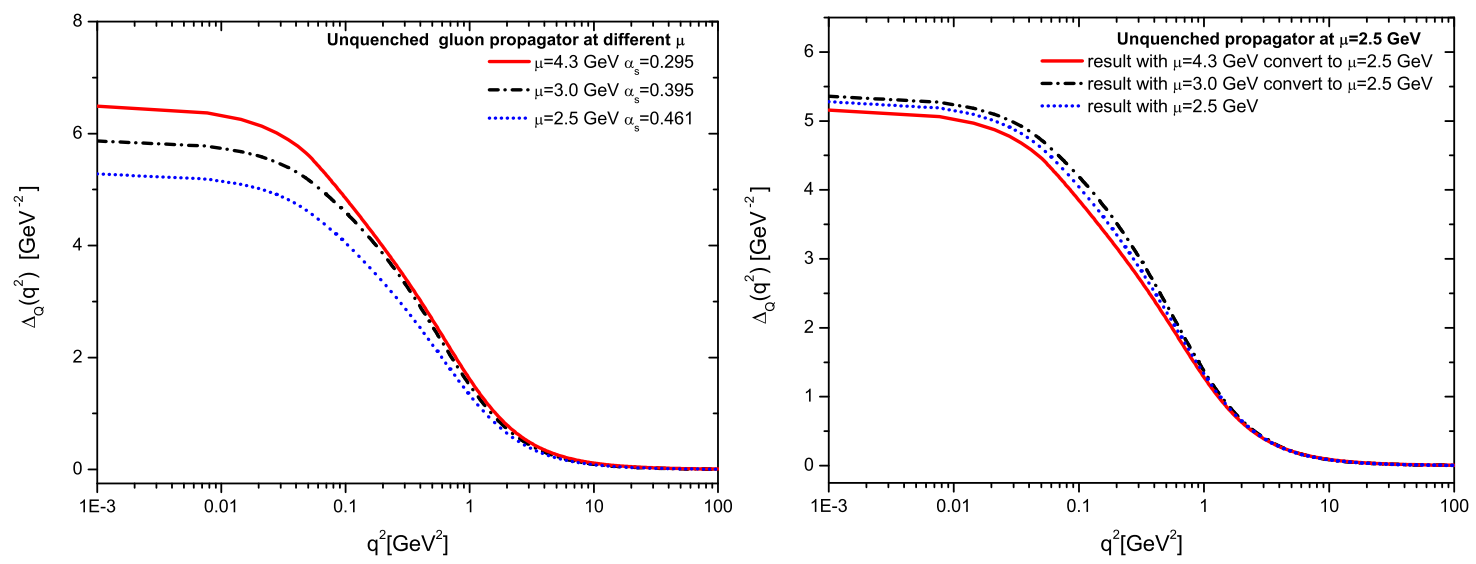

FIG. 16: Left panel: The $n_{f}=2$ unquenched gluon propagator renormalized at different values of $\mu$ and $\alpha_{s}: \mu=4.3 \mathrm{GeV}$ and $\alpha_{s}=0.295$ (solid red curve), $\mu=3.0 \mathrm{GeV}$ and $\alpha_{s}=0.395$ (dash-dotted black curve), $\mu=2.5 \mathrm{GeV}$ and $\alpha_{s}=0.461$ (dotted blue curve). Right panel: All curves showed on the left panel renormalized at the same point $\mu=2.5 \mathrm{GeV}$ using the Eq. (4.1).

different values of $\mu$ is the multiplicative renormalizability, which allows one to connect a set of data renormalized at $\mu$ with a corresponding set renormalized at $\nu$, through the relation

$$
\Delta_{Q}\left(q^{2}, \mu^{2}\right)=\frac{\Delta_{Q}\left(q^{2}, \nu^{2}\right)}{\mu^{2} \Delta_{Q}\left(\mu^{2}, \nu^{2}\right)} .
$$

On the right panel of Fig. 16 we check how $\Delta_{Q}\left(q^{2}\right)$ behaves under changes of $\mu$ using Eq. (4.1). Evidently, multiplicative renormalizability would require that the three curves lie on top of each other; however we see that there is a minor difference between them (at the $4 \%$ level), whose origin might be related to the fact that, as discussed in the previous section, in our computation the renormalization procedure was carried out subtractively instead of multiplicatively.

\section{Comparison with the lattice data}

In this final subsection we carry our a comparison between the results we found for the gluon dressing function, $\mathcal{Z}_{Q}\left(q^{2}\right)$, and the data obtained from the unquenched lattice simulation of Ref. [6]. We remind the reader that, according to the convention introduced below Eq. (3.7), we will denote by $M$ (with the appropriate flavor index) the value of the corresponding running quark mass $\mathcal{M}\left(p^{2}\right)$ at $p^{2}=0$.

On the left panel of Fig. 17, we show the $2+1$ flavor QCD lattice data renormalized at $\mu=4.3 \mathrm{GeV}$ (gray open circles), together with the results obtained applying our calculational 

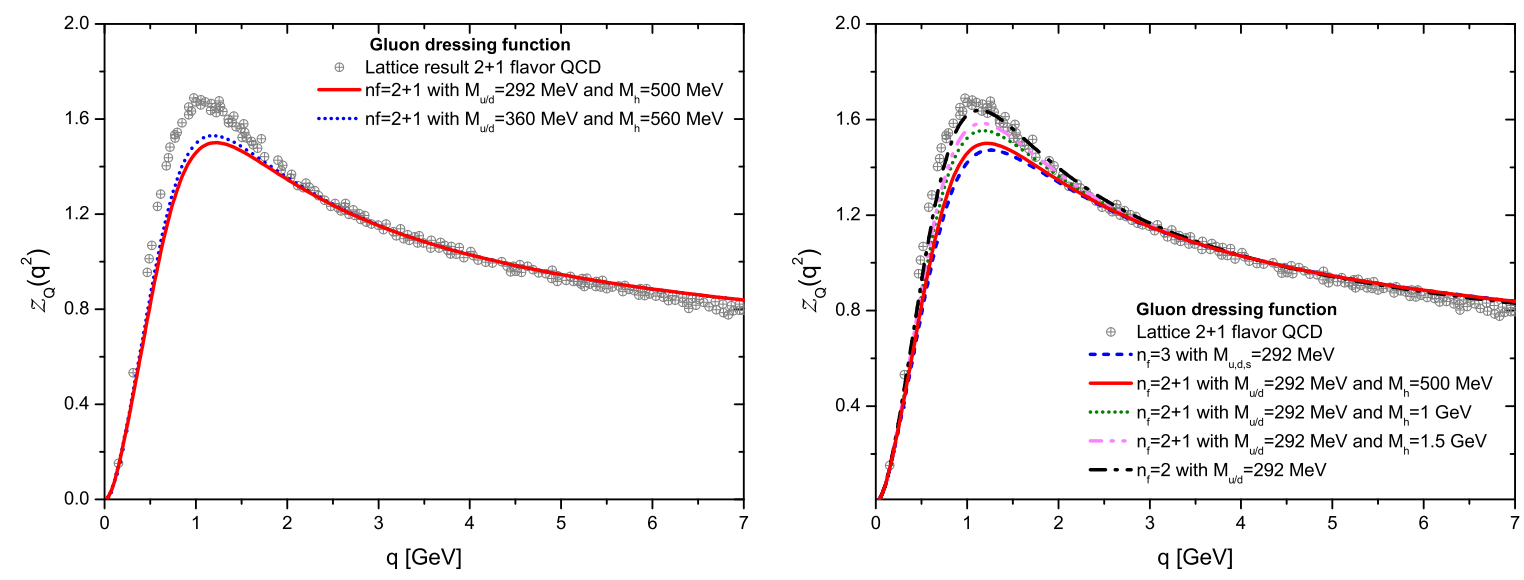

FIG. 17: Left panel: The unquenched gluon dressing function $\mathcal{Z}_{Q}\left(q^{2}\right)$ obtained in the Ref. [6] (open gray circles) together with the one-loop result for two light quarks with $M_{u / d}=292 \mathrm{MeV}$, and one heavier with $M_{s}=500 \mathrm{MeV}$ (continuous red curve) and the case where $M_{u / d}=360 \mathrm{MeV}$ and $M_{s}=560 \mathrm{MeV}$ (dotted blue curve). Right panel: The one-loop $\mathcal{Z}_{Q}\left(q^{2}\right)$ for $n_{f}=2+1$ flavors. The light quarks have constant masses of $M_{u / d}=292 \mathrm{MeV}$ while for the heavier quark: $M_{h}=292 \mathrm{GeV}$ (dashed blue curve), $M_{h}=500 \mathrm{MeV}$ (solid red curve), $M_{h}=1.0 \mathrm{GeV}$ (dotted green curve) and $M_{h}=1.5 \mathrm{GeV}$ (dashed with two dots magenta curve).

procedure, with two light quarks of mass $M_{u / d}=292 \mathrm{MeV}$ and one heavier of $M_{h}=500 \mathrm{MeV}$ (solid red curve). It is important to mention that the above ranges of quark masses are consistent with the values generally employed in phenomenological calculations [61, 62].

We clearly see that the overall shape of the calculated curves display a nice agreement with the data in a sizable range of momenta. The region where the difference between the curves is more pronounced is around $q=1.25 \mathrm{GeV}$, exactly where the peak of $\mathcal{Z}_{Q}\left(q^{2}\right)$ is located. However, observe that, even in this least favorable region, the difference between these curves is no greater than $10 \%$.

In addition, notice that on the same plot we display also the case where $M_{u / d}=360 \mathrm{MeV}$ and $M_{h}=560 \mathrm{MeV}$ (dashed blue curve). The way this latter set of mass values (and corresponding quark propagators) are obtained is by solving the quark gap equation using suitable values for the current masses. More specifically, for the light quarks (up/down) we use a current mass of $14 \mathrm{MeV}$, while for the heavier one (strange) we use $68 \mathrm{MeV}$, in agreement with the values quoted in Ref. [6]. Observe that the dotted blue curve indicates that the increase of masses produces a slight change in the peak of the dressing function. 
It would be instructive to analyze how different choices for $M_{h}$ modify the form $\mathcal{Z}_{Q}\left(q^{2}\right)$ shown on the left panel of Fig. 17, As we have already shown on the right panel of Fig. 15, the gluon propagator becomes progressively suppressed as the quark mass increases. It is therefore natural to expect that the gluon dressing function will be also affected by different choices of masses.

On the right panel of the Fig. [17, we show how $\mathcal{Z}_{Q}\left(q^{2}\right)$ varies with $M_{h}$. In all curves the light quarks have constant masses of $M_{u / d}=292 \mathrm{MeV}$, whereas for the heavier quark we use: $M_{h}=292 \mathrm{GeV}$ (dashed blue curve), $M_{h}=500 \mathrm{MeV}$ (solid red curve), $M_{h}=1.0 \mathrm{GeV}$ (dotted green curve) and $M_{h}=1.5 \mathrm{GeV}$ (dashed with two dots magenta curve).

As we clearly see, the peak of $\mathcal{Z}_{Q}\left(q^{2}\right)$ becomes more pronounced as we increase the value of $M_{h}$. Notice that the case where $M_{h}=1.5 \mathrm{GeV}$ (dashed with two dots magenta curve) is much closer to the lattice data (open gray circles). In addition, if we keep increasing the heavy quark mass gradually, the observed trend of the results is to move progressively closer to the case where only two quarks are active (dash-dotted black curve), thus confirming the notion of decoupling of heavy flavors. To avoid any possible confusion caused by the striking proximity of the $n_{f}=2$ curve to the lattice data, we reiterate that the real comparison between the $(2+1)$ data and the corresponding $(2+1)$ SDE result is given on the left panel. Evidently, the analogous comparison of the $n_{f}=2$ curve would require a corresponding set of lattice data, not available to us at present.

\section{CONCLUSIONS}

In this article we have presented a general method for estimating the effects that the "unquenching" induces on the (IR finite) gluon propagator, in the Landau gauge. The basic assumption of the method followed has been that the main bulk of the effect originates from the "one-loop dressed" quark diagram, while the rest of the contributions is considered to be subleading. We have restricted the applicability of this approach to a small number of quark families $(n=1,2,3)$, where we assume the presence of the quarks does not alter

qualitatively the behavior of the quenched propagator. In particular, we expect that the crucial property of IR finiteness will persist, i.e., the gluon mass generating mechanism will not be distorted by the inclusion of a few quark families. In fact, throughout our analysis we use the quenched gluon propagator obtained in $S U(3)$ lattice simulations as our point of 
reference, and estimate the deviations induced to it by the quarks.

The nonperturbative calculation of the quark loop proceeds by means of two suitable Ansätze for the fully dressed quark-gluon vertex $\widehat{\Gamma}_{\mu}$, enforcing the exact transversality of the resulting contribution. The use of the PT-BFM formalism simplifies the form of these Ansätze considerably, due to the "abelianization" that it induces, given that the corresponding Green's functions, when contracted with respect to the momentum carried by the background leg, satisfy linear ghost free WIs instead of the usual nonlinear STIs. This fact, in turn, avoids the explicit reference to the quark-ghost kernel, which appears in the standard STI satisfied by the conventional quark-gluon vertex $\Gamma_{\mu}$ [the $H$ auxiliary function of Eq. (3.3)]. Of course, one cannot completely eliminate any dependence on $H$, for the simple reason that it affects the quark gap equation that determines the quantities $A(p)$ and $B(p)$, namely the nonperturbative Dirac components of the quark propagator; this happens because, as explained in [31], the quark-gluon vertex entering in the gap equation is $\Gamma_{\mu}$ and not $\widehat{\Gamma}_{\mu}$. Given that the structure of the quark-ghost kernel is largely unexplored (for an SD estimate of one of its form-factors, see [31]]), reducing the dependence of the answer on it is clearly advantageous.

The main results of our study is that the inclusion of the quark loop(s) induces a suppression in the intermediate and IR momentum regions, with respect to the quenched case. As emphasized in the main text, the actual saturation point of the unquenched propagator, i.e., the value $\Delta_{Q}(0)$, normally associated with the IR value of the dynamical gluon mass, $m^{2}(0)$, is not possible to determine at present, despite the fact that the quark-loop contribution to the corresponding gluon self-energy vanishes at $q^{2}=0$, by virtue of a powerful identity. The reason is that the momentum evolution of the gluon mass depends (in a yet not fully determined way) on the structure of the gluon propagator through the entire range of physical momenta; thus, the suppression of the propagator due to the inclusion of the quarks is expected to modify the value of $m^{2}(0)$. In this work we have adopted a simple hand-waving approach for estimating $\Delta_{Q}(0)$. Specifically, given that the unquenched propagator in the IR and intermediate regions is consistently below the corresponding quenched curve, we have simply extrapolated towards the point $q^{2}=0$. In practice, the outcome of this simple procedure depends to some extent on the extrapolation details (in particular, what one considers as the last "faithful" point), and therefore one can only determine a certain range of "reasonable" values for $\Delta_{Q}(0)$. 
The uncertainty associated with the determination of the saturation point is practically eradicated if one considers instead of the gluon propagator its corresponding dressing function. This latter quantity, when compared to the corresponding dressing function of the quenched lattice propagator, clearly demonstrates the aforementioned suppression in the IR and intermediate regions induced by the inclusion of the quarks. The unquenched dressing function obtained through our procedure appears to be in rather good agreement with the lattice results available in the literature.

There are certain theoretical improvements, which, if successfully implemented, would put the proposed approach on a more solid ground. To begin with, it is clear that the full SDE treatment of the problem at hand would entail the simultaneous treatment of a complicated set of coupled integral equations, in the spirit presented in [38, 39], in the context of the scaling solutions. This type of global treatment appears to be beyond our present calculational powers, mainly due to the plethora of additional technical complications intrinsic to the massive solutions. Instead, we have adopted a step-by-step procedure; for example, the quark-gap equation has been solved "in isolation", and the obtained solutions have been fed into the equations determining the quark-loop, and so on. To be sure, this latter procedure might interfere with the nonlinear propagation of certain effects, leading to the corresponding amplification or suppression of various features, and may require additional refinements.

The renormalization properties of the relevant integral equations constitute a commonly known source of theoretical uncertainty, due to the mishandling of the overlapping divergences induced by the well-known intrinsic ambiguity of the gauge-technique, related to the unspecified transverse (automatically conserved) part of the vertices. In particular, the $\mathrm{BC}$ and $\mathrm{CP}$ expressions employed here for the quark-gluon vertex do not fully respect the property of multiplicative renormalizability, which, in turn, leads to dependences on the renormalization point that are not always in accordance with those dictated by the renormalization group. The propagation of such discrepancies to our predictions has been studied numerically, and appears to be relatively suppressed. However, more work is clearly needed in order to eliminate the spurious $\mu$-dependences. In this vain, it would be interesting, albeit logistically cumbersome, to explore the effects that other forms of the quark-gluon vertex might have on our predictions, such as those reported in [63, 64].

Finally, the reliable calculation of the saturation point $\Delta_{Q}(0)$ mentioned above hinges 
explicitly on the derivation of a fully self-consistent integral equation, that would determine the momentum evolution of the dynamical gluon mass, both in the quenched case and in the presence of quarks. The derivation of such a complete equation is conceptually and technically rather non-trivial, and is the subject of an ongoing investigation, whose results will be hopefully presented soon.

\section{Acknowledgments}

The research of J. P. is supported by the Spanish MEYC under grant FPA2011-23596. The work of A.C.A is supported by the Brazilian Funding Agency CNPq under the grant 305850/2009-1 and project 474826/2010-4 .

\section{Appendix A: The perturbative One-loop case}

The text-book perturbative calculation of diagram $a_{11}$ yields (with $d_{f}=1 / 2$ )

$$
\widehat{X}^{[1]}\left(q^{2}\right)=-\frac{2 g^{2}}{d-1} \int_{k} \frac{d M^{2}-(d-2)\left(k^{2}+k \cdot q\right)}{\left(k^{2}-M^{2}\right)\left[(k+q)^{2}-M^{2}\right]} .
$$

where $M$ denotes a constant (momentum-independent) mass. Note that the "hat" in this case is redundant, because, at one loop, the conventional and BFM results coincide. The result of Eq. (A1) may be directly recovered from the general case presented in section [II, by setting $\mathcal{M}(p)=M, A(p)=1, L_{1}=1, L_{2}=L_{3}=0$ in Eqs.(3.14) and (3.15).

It is elementary to establish that

$$
\widehat{X}^{[1]}(0)=0
$$

by virtue of the basic identity

$$
\int_{k} \frac{k^{2}}{\left(k^{2}-M^{2}\right)^{2}}=\frac{d}{2} \int_{k} \frac{1}{k^{2}-M^{2}}
$$

or, equivalently,

$$
2 M^{2} \int_{k} \frac{1}{\left(k^{2}-M^{2}\right)^{2}}=(d-2) \int_{k} \frac{1}{k^{2}-M^{2}},
$$

whose validity may be easily verified following the integration rules of dimensional regularization. These exact same identities appear in the standard one-loop calculation of the 
photon vacuum polarization, both in normal QED and in scalar QED, and enforces the masslessness of the photon [54].

The property of (A2) becomes manifest through the use of (A4), which allows one to cast (A1) into the form

$$
\widehat{X}^{[1]}\left(q^{2}\right)=-\frac{g^{2}}{d-1}\left\{(d-2) q^{2} I\left(q^{2}\right)+4 M^{2}\left[I\left(q^{2}\right)-I(0)\right]\right\},
$$

where

$$
I\left(q^{2}\right)=\int_{k} \frac{1}{\left(k^{2}-M^{2}\right)\left[(k+q)^{2}-M^{2}\right]},
$$

or, equivalently, defining

$$
u^{2}\left(q^{2}\right) \equiv q^{2} x(x-1)+M^{2}
$$

we have

$$
\widehat{X}^{[1]}\left(q^{2}\right)=-\frac{g^{2}}{d-1}\left\{(d-2) q^{2} I\left(q^{2}\right)-i \frac{M^{2}}{4 \pi^{2}} \int_{0}^{1} \mathrm{~d} x \ln \frac{u^{2}\left(q^{2}\right)}{M^{2}}\right\} .
$$

Finally, the renormalized expression for $\widehat{X}^{(1)}\left(q^{2}\right)$ in the MOM scheme is given by

$$
\widehat{X}_{R}^{[1]}\left(q^{2}\right)=\widehat{X}^{[1]}\left(q^{2}\right)-\frac{q^{2}}{\mu^{2}} \widehat{X}^{[1]}\left(\mu^{2}\right)
$$

giving as a result

$$
\widehat{X}_{R}^{[1]}\left(q^{2}\right)=\frac{i \alpha_{s}}{6 \pi}\left\{q^{2} \int_{0}^{1} \mathrm{~d} x \ln \frac{u^{2}\left(q^{2}\right)}{u^{2}\left(\mu^{2}\right)}+2 M^{2}\left[\int_{0}^{1} \mathrm{~d} x \ln \frac{u^{2}\left(q^{2}\right)}{M^{2}}-\frac{q^{2}}{\mu^{2}} \int_{0}^{1} \mathrm{~d} x \ln \frac{u^{2}\left(\mu^{2}\right)}{M^{2}}\right]\right\} .
$$

Evidently, for $q^{2}$ and $\mu^{2}$ much larger than $M^{2}$, one obtains the standard logarithmic correction

$$
\widehat{X}_{R}^{[1]}\left(q^{2}\right)=\frac{i \alpha_{s}}{6 \pi} q^{2} \ln \left(-q^{2} / \mu^{2}\right)
$$

[1] A. Cucchieri and T. Mendes, PoS LAT2007, 297 (2007).

[2] A. Cucchieri and T. Mendes, Phys. Rev. Lett. 100, 241601 (2008).

[3] A. Cucchieri and T. Mendes, Phys. Rev. D 81, 016005 (2010).

[4] A. Cucchieri and T. Mendes, PoS LATTICE2010, 280 (2010).

[5] W. Kamleh, P. O. Bowman, D. B. Leinweber, A. G. Williams and J. Zhang, Phys. Rev. D 76, 094501 (2007). 
[6] P. O. Bowman, U. M. Heller, D. B. Leinweber, M. B. Parappilly, A. Sternbeck, L. von Smekal, A. G. Williams and J. -b. Zhang, Phys. Rev. D 76, 094505 (2007).

[7] I. L. Bogolubsky, E. M. Ilgenfritz, M. Muller-Preussker and A. Sternbeck, PoS LATTICE, 290 (2007).

[8] I. L. Bogolubsky, E. M. Ilgenfritz, M. Muller-Preussker and A. Sternbeck, Phys. Lett. B 676, $69(2009)$.

[9] O. Oliveira, P. J. Silva, Phys. Rev. D79, 031501 (2009).

[10] O. Oliveira and P. J. Silva, PoS LAT2009, 226 (2009).

[11] R. Alkofer, L. von Smekal, Phys. Rept. 353, 281 (2001).

[12] C. S. Fischer, J. Phys. G G32, R253-R291 (2006).

[13] A. C. Aguilar and J. Papavassiliou, JHEP 0612, 012 (2006).

[14] D. Binosi and J. Papavassiliou, Phys. Rev. D 77(R), 061702 (2008).

[15] A. C. Aguilar, D. Binosi and J. Papavassiliou, Phys. Rev. D 78, 025010 (2008).

[16] D. Binosi and J. Papavassiliou, Phys. Rept. 479, 1-152 (2009).

[17] J. Rodriguez-Quintero, Phys. Rev. D83, 097501 (2011).

[18] J. Rodriguez-Quintero, JHEP 1101, 105 (2011).

[19] Ph. Boucaud, M. E. Gomez, J. P. Leroy, A. Le Yaouanc, J. Micheli, O. Pene, J. RodriguezQuintero, Phys. Rev. D82, 054007 (2010).

[20] Ph. Boucaud, F. De Soto, J. P. Leroy, A. Le Yaouanc, J. Micheli, O. Pene and J. RodriguezQuintero, Phys. Rev. D 79, 014508 (2009).

[21] P. Boucaud, J-P. Leroy, A. L. Yaouanc, J. Micheli, O. Pene and J. Rodriguez-Quintero, JHEP 0806, 012 (2008).

[22] C. S. Fischer, A. Maas and J. M. Pawlowski, Annals Phys. 324, 2408 (2009).

[23] A. P. Szczepaniak and H. H. Matevosyan, Phys. Rev. D 81, 094007 (2010).

[24] A. C. Aguilar and A. A. Natale, JHEP 0408, 057 (2004).

[25] D. Dudal, J. A. Gracey, S. P. Sorella, N. Vandersickel and H. Verschelde, Phys. Rev. D 78, 065047 (2008).

[26] D. Dudal, O. Oliveira, N. Vandersickel, Phys. Rev. D81, 074505 (2010).

[27] D. Dudal, S. P. Sorella and N. Vandersickel, Phys. Rev. D 84, 065039 (2011).

[28] K. -I. Kondo, Phys. Rev. D 84, 061702 (2011).

[29] A. C. Aguilar, D. Binosi and J. Papavassiliou, Phys. Rev. D 81, 125025 (2010). 
[30] A. C. Aguilar, D. Binosi and J. Papavassiliou, JHEP 1007, 002 (2010).

[31] A. C. Aguilar and J. Papavassiliou, Phys. Rev. D 83, 014013 (2011).

[32] A. C. Aguilar, D. Binosi and J. Papavassiliou, JHEP 1201, 050 (2012).

[33] A. Cucchieri, D. Dudal, T. Mendes and N. Vandersickel, arXiv:1111.2327 [hep-lat].

[34] D. Dudal, N. Vandersickel, A. Cucchieri and T. Mendes, PoS QCD -TNT-II, 015 (2011).

[35] J. I. Skullerud, P. O. Bowman, A. Kizilersu, D. B. Leinweber and A. G. Williams, JHEP 0304, 047 (2003).

[36] A. Cucchieri, A. Maas and T. Mendes, Phys. Rev. D 77, 094510 (2008).

[37] P. .Boucaud, D. Dudal, J. P. Leroy, O. Pene and J. Rodriguez-Quintero, JHEP 1112, 018 (2011).

[38] C. S. Fischer and R. Alkofer, Phys. Rev. D 67, 094020 (2003).

[39] C. S. Fischer, P. Watson and W. Cassing, Phys. Rev. D 72, 094025 (2005).

[40] J. M. Cornwall, Phys. Rev. D 26, 1453 (1982).

[41] J. M. Cornwall and J. Papavassiliou, Phys. Rev. D 40, 3474 (1989).

[42] D. Binosi and J. Papavassiliou, Phys. Rev. D 66(R), 111901 (2002).

[43] D. Binosi and J. Papavassiliou, J. Phys. G 30, 203 (2004).

[44] See, e.g., L. F. Abbott, Nucl. Phys. B 185, 189 (1981), and references therein.

[45] D. Binosi and J. Papavassiliou, JHEP 0811, 063 (2008).

[46] W. J. Marciano and H. Pagels, Phys. Rept. 36, 137 (1978).

[47] J. S. Ball, T. -W. Chiu, Phys. Rev. D22, 2542 (1980).

[48] D. C. Curtis and M. R. Pennington, Phys. Rev. D 42, 4165 (1990).

[49] P. A. Grassi, T. Hurth and A. Quadri, Phys. Rev. D 70, 105014 (2004).

[50] A. C. Aguilar, D. Binosi and J. Papavassiliou, JHEP 0911, 066 (2009).

[51] A. C. Aguilar, D. Binosi, J. Papavassiliou and J. Rodriguez-Quintero, Phys. Rev. D 80, 085018 (2009).

[52] A. Sternbeck, hep-lat/0609016.

[53] A. C. Aguilar, D. Binosi and J. Papavassiliou, Phys. Rev. D 84, 085026 (2011).

[54] A. C. Aguilar and J. Papavassiliou, Phys. Rev. D 81, 034003 (2010).

[55] A. C. Aguilar, D. Ibanez, V. Mathieu and J. Papavassiliou, Phys. Rev. D 85, 014018 (2012).

[56] L. Del Debbio, PoS LATTICE 2010, 004 (2010).

[57] X. Cheng and E. T. Tomboulis, PoS QCD -TNT-II, 046 (2011). 
[58] A. I. Davydychev, P. Osland and L. Saks, Phys. Rev. D 63, 014022 (2001).

[59] C. D. Roberts and A. G. Williams, Prog. Part. Nucl. Phys. 33, 477 (1994).

[60] P. A. Grassi, T. Hurth and M. Steinhauser, Annals Phys. 288, 197 (2001).

[61] P. Maris and C. D. Roberts, Phys. Rev. C 56, 3369 (1997).

[62] B. El-Bennich, G. Krein, L. Chang, C. D. Roberts and D. J. Wilson, Phys. Rev. D 85, 031502 (2012).

[63] A. Kizilersu and M. R. Pennington, Phys. Rev. D 79, 125020 (2009); A. Bashir, A. Kizilersu and M. R. Pennington, Phys. Rev. D 57, 1242 (1998).

[64] A. Bashir, R. Bermudez, L. Chang and C. D. Roberts, arXiv:1112.4847 [nucl-th]. 